\title{
DISCRIMINATIVE SPARSE CODING IN THE ANALYSIS OF ELECTROCARDIOGRAM DURING VENTRICULAR ARRHYTHMIAS
}

\author{
by \\ Iman Kalaji \\ B.Eng., Ryerson University, June 2012
}

\begin{abstract}
A thesis
presented to Ryerson University

in partial fulfillment

of the requirements for the degree of

Master of Applied Science in the Program of

Electrical and Computer Engineering
\end{abstract}

Toronto, Ontario, Canada

(C)Iman Kalaji 2015 
I hereby declare that I am the sole author of this thesis. This is a true copy of the thesis, including any required final revisions, as accepted by my examiners.

I authorize Ryerson University to lend this thesis to other institutions or individuals for the purpose of scholarly research.

I further authorize Ryerson University to reproduce this thesis by photocopying or by other means, in total or in part, at the request of other institutions or individuals for the purpose of scholarly research.

I understand that my thesis may be made electronically available to the public. 


\begin{abstract}
Discriminative Sparse Coding in the Analysis of Electrocardiogram

During Ventricular Arrhythmias,

Master of Applied Science, 2015,

Iman Kalaji,

Department of Electrical and Computer Engineering, Ryerson University.
\end{abstract}

Abnormalities in the rhythmic electromechanical contractions of the heart results in cardiac arrhythmias. When these abnormalities rise from the ventricles of the heart, they are classified as ventricular arrhythmias. The two major types of ventricular arrhythmias are ventricular tachycardia (VT) and ventricular fibrillation (VF). Ventricular fibrillation is the most dangerous among the two arrhythmias, that usually leads to sudden cardiac death if not treated immediately. Annually about 40,000 sudden cardiac deaths are reported in Canada. Due to high mortality rate and serious impact on quality of life, researchers have been focusing on characterizing ventricular arrhythmias that may lead to delivering optimized treatment options in improving the survival rates.

In this thesis two major types of ventricular arrhythmias were analyzed and quantified by performing a discriminative sparse coding analysis called label consistent K-SVD using timefrequency dictionaries that are well localized in time and frequency domains. The analyzed signals were 670 ECG ventricular arrhythmia segments from 33 patients extracted from the Malignant Ventricular Ectopy and Creighton University Tachy-Arrhythmia databases. Using the LCKSVD dictionary learning approach, an overall maximum classification accuracy of $73.3 \%$ was achieved with a hybrid optimized wavelet dictionary. Based on the comparative analysis, the trained (learned) dictionaries yielded better performance than the untrained dictionaries. The results indicate that discriminative sparse coding approach has greater potential in extracting signal adaptive and morphologically discriminative time-frequency structures in studying ventricular arrhtyhmias. 


\section{Acknowledgments}

I would like to express my sincere gratitude to my supervisor Dr. K. Umapathy for the continuous support, guidance, and motivation. His guidance helped me in all the time of research, I could not have imagined having a better adviser and mentor for my master study. I also thankfully acknowledge Mr. Balasundaram for providing me with his help and feedback whenever I needed it. Last but not least I would like to thank my parents, who inspired me for higher studies and have supported me throughout the entire process, both by keeping me motivated and helping me putting the pieces together. I will be grateful forever for your love and support, I could not have done it without you. The most special thank goes to my partner and friend, my husband Mohammad for his love and support during the difficult times and for always being so patient with me. Love you. 
To my beloved parents,

to my husband,

and to my best friend my brother. 


\section{Contents}

Author's Declaration

$\begin{array}{lll}\text { Abstract } & \text { iii }\end{array}$

Acknowledgments $\quad$ iv

Dedication $\quad$ v

List of Tables

$\begin{array}{ll}\text { List of Figures } & \text { ix }\end{array}$

1 Introduction $\quad 1$

1.1 Electrophysiology of the Cardiovascular System . . . . . . . . . . . . . . 1

1.2 Ventricular Arrhythmias . . . . . . . . . . . . . . . . . 3

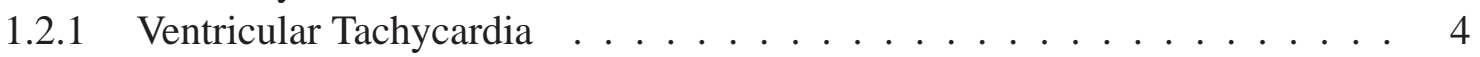

1.2.2 Ventricular Fibrillation . . . . . . . . . . . . . . . . 6

1.3 ECG Analysis During Ventricular Arrhythmias . . . . . . . . . . . . . 6

1.3.1 Time-Domain Analysis . . . . . . . . . . . . . . . . 6

1.3.2 Frequency-Domain Analysis .................... 7

1.3.3 Time-Frequency Domain Analysis . . . . . . . . . . . . . . . . 8

1.4 Motivation . . . . . . . . . . . . . . . . . . . . 9

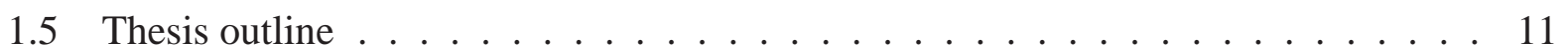

2 Methods Background $\quad 13$

2.1 Time-Frequency Domain Analysis . . . . . . . . . . . . . . . . . . . 13

2.2 Time-Frequency Dictionaries . . . . . . . . . . . . . . . . . . . . . . . . . . . . 17

2.2 .1 Parametric Dictionaries . . . . . . . . . . . . . . . 18

2.2.2 Learned Dictionaries . . . . . . . . . . . . . . . . . 20

2.2.3 Label-Consistent K-SVD . . . . . . . . . . . . . . . . . . . . . . . . . . . . . . . . . . . . . .

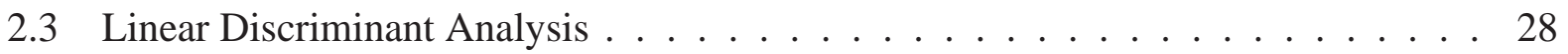

2.4 Cross Validation . . . . . . . . . . . . . . . . . . . . 30

2.5 Background Summary . . . . . . . . . . . . . . . . 31 
$3 \quad$ LC-KSVD Parameters For Ventricular Arrhythmia Analysis 32

3.1 Database and Pre-processing . . . . . . . . . . . . . . . . . . . . 32

3.2 Dictionary Selection . . . . . . . . . . . . . . . . . 33

$3.2 .1 \quad$ Gabor Dictionary . . . . . . . . . . . . . . . . . . . . 34

3.2 .2 Wavelet Dictionaries . . . . . . . . . . . . . . . . . . . 35

3.2 .3 Optimal Dictionary . . . . . . . . . . . . . . . . . . . 37

3.3 Choice of Optimal Parameters . . . . . . . . . . . . . . . . . . . . . 37

3.4 Chapter 3 Summary . . . . . . . . . . . . . . . . . . . . . . . . . 41

4 Feature Extraction and Pattern Classification 44

4.1 Features/ Feature Space . . . . . . . . . . . . . . . . . . . . . . . . . . . 44

4.2 LC-KSVD analysis on Arrhythmic Signals . . . . . . . . . . . . . . . . . 45

4.2.1 Results Using Optimized Dictionaries . . . . . . . . . . . . . . . . . 46

4.2 .2 Results Using Full Dictionary . . . . . . . . . . . . . . . . . . . . 54

4.3 Results Summary . . . . . . . . . . . . . . . . . . . . . . . . . . 59

4.4 Chapter 4 Summary . . . . . . . . . . . . . . . . . . . . . . . . 60

5 Conclusions and Future Work $\quad 61$

5.1 Summary and Conclusions $\ldots \ldots \ldots$. . . . . . . . . . . . . 61

5.2 Future Work . . . . . . . . . . . . . . . . . . . . . . . 62

$\begin{array}{lr}\text { A Abbreviations } & 63\end{array}$

B Optimization Results $\quad 64$

B.1 Sample optimization results using different $\alpha$ for a subset of data using Gabor and Sym4 dictionary . . . . . . . . . . . . . . . . . . . . . . . 64

B.2 Sample optimization results using different $\alpha$ and $\beta$ for a subset of data using Gabor and Sym4 dictionary . . . . . . . . . . . . . . . . . . . 64

$\begin{array}{ll}\text { Bibliography } & \mathbf{6 6}\end{array}$ 


\section{List of Tables}

3.1 Classification accuracy and reconstruction error for different $\alpha$ parameters for 2000 iterations of LC-KSVD . . . . . . . . . . . . . . . . . . . . 40

4.1 Average classification accuracy for different combinations of the optimized dictionaries for 2000 iterations of LC-KSVD . . . . . . . . . . . . . . . . . 48

4.2 Confusion matrix of discriminating VF and VT signals using optimized Symlet4-Wpsym4 dictionaries of LC-KSVD . . . . . . . . . . . . . . . . . 48

4.3 Range of the 5-folds confusion matrix of discriminating VF and VT signals using optimized Symlet4-Wpsym4 dictionaries of LC-KSVD . . . . . . . . . . . . . 49

4.4 The Classification accuracy for the five folds data sets using LC-KSVD1 and LC-

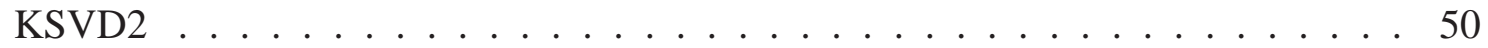

4.5 The Classification accuracy for the five folds data sets using the optimized trained and non-trained Symlet4-Wpsym4 dictionaries _. . . . . . . . . . . . 50

4.6 Average classification accuracy for different combinations of the full dictionaries for 2000 iterations of LC-KSVD . . . . . . . . . . . . . . . . . 57

4.7 Confusion matrix of discriminating VF and VT signals using full Gabor-Wpsym4 dictionaries of LC-KSVD . . . . . . . . . . . . . . . . . . . . 57

4.8 Range of the 5-folds confusion matrix of discriminating VF and VT signals using full Gabor-Wpsym4 dictionary of LC-KSVD . . . . . . . . . . . . . . . 58

4.9 The Classification accuracy for the four data sets using non-trained and LC-KSVD trained full Gabor-Wpsymlet 4 dictionary $\ldots \ldots \ldots . \ldots . \ldots 58$

B.1 Classification accuracy and error 1 for different $\alpha$ parameters for 2000 iterations of LC-KSVD . . . . . . . . . . . . . . . . . . . . . . . . . 64

B.2 Total error 2 for different combinations of $\alpha$ and $\beta$ parameters for 2000 iterations of LC-KSVD . . . . . . . . . . . . . . . . . . . . . . . 65

B.3 Classification accuracy for different combinations of $\alpha$ and $\beta$ parameters for 2000 iterations of LC-KSVD . . . . . . . . . . . . . . . . . . . . 65 


\section{List of Figures}

1.1 The anatomy of the heart $\subset$ McGraw-Hill $[1] \ldots \ldots \ldots 2$

1.2 The electrical conduction pathway of the heart $(\mathrm{C}$ McGraw-Hill [1] . . . . . . . . 3

1.3 The electrical conduction of normal sinus rhythm @ CMcGraw-Hill [1] . . . . . . 4

1.4 Three second ventricular tachycardia (top), three second ventricular fibrillation

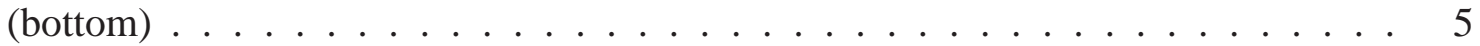

1.5 Thesis outline . . . . . . . . . . . . . . . . . . . . . . . . . 11

2.1 a) Coverage of the time-frequency plane for the STFT. b) Coverage of the timefrequency plane for the wavelet. c) Corresponding basis function of the STFT. d) Corresponding basis function of the wavelets $[2] \ldots \ldots \ldots$

2.2 Gabor Atom . . . . . . . . . . . . . . . . . . . . . . . . . . . . . . . . . . . . . . 19

$2.3 \mathrm{M}$ fold cross validation structure $\ldots \ldots \ldots$. . . . . . . . . . . . . 30

3.1 Sample signals of $\mathrm{VF}$ and $\mathrm{VT} \ldots \ldots \ldots \ldots \ldots \ldots$

3.2 Four different Gabor atoms from the generated dictionary of different translation and scales . . . . . . . . . . . . . . . . . . 35

3.3 Four different Wavelet atoms from the generated dictionaries . . . . . . . . . . 36

3.4 Matching pursuit on VF signal (Top) and it's captured energy (bottom) using Dictionary $1 \ldots \ldots \ldots \ldots \ldots \ldots \ldots$

3.5 Matching pursuit on VT signal (Top) and it's captured energy (bottom) using Dic-

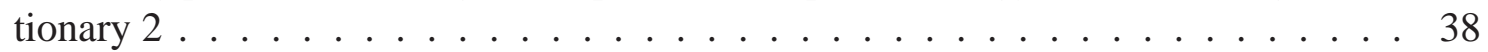

3.6 Matching pursuit on VF signal (Top) and it's captured energy (bottom) using Dictionary $3 \ldots \ldots \ldots$. . . . . . . . . . . . . . . . . . . 39

3.7 Classification accuracies using different $\alpha$ values . . . . . . . . . . . . . . 41

3.8 Total error 1 using different $\alpha$ values . . . . . . . . . . . . . . . . . . 42

3.9 Boxplot of the classification accuracy ranges using different $\alpha$ and $\beta$ values . . . . 42

3.10 Boxplot of the total error 2 ranges using different $\alpha$ and $\beta$ values . . . . . . . . 43

4.1 An example of feature space of the estimated score feature, where blue pluses represents VF tested signals and red circles represents VT tested signals . . . . . 45

4.2 Flow chart of the results analysis . . . . . . . . . . . . . . . . . . . 47

4.3 Boxplot of classification accuracies of LC-KSVD1 and LC-KSVD2 . . . . . . . 49 
4.4 Boxplot of the classification accuracy of the five fold data sets of VF and VT using optimized trained and non-trained Symlet4-Wpsymlet4 dictionaries . . . . . . . 51

4.5 Examples of Symlet4-Wpsymlet4 trained dictionary atoms . . . . . . . . . . . 51

4.6 Boxplot of the classification accuracy of the five fold data sets of VF and VT using optimized trained and non-trained Gabor-Wpsymlet4 dictionaries . . . . . . . 52

4.7 Boxplot of the classification accuracy of the five fold data sets of VF and VT using optimized trained and non-trained Gabor-Symlet 4 dictionaries . . . . . . . . . . 53

4.8 Classification accuracy using different sizes of the Gabor-Wpsym4 dictionary . . . 54

4.9 Computational time of one fold using LC-KSVD with respect to different sizes of the Gabor-Wpsym4 dictionary . . . . . . . . . . . . . . . . . . 55

4.10 Comparing different types of classifiers for classifying the feature score of the LCKSVD . . . . . . . . . . . . . . . . . . . 56

4.11 Boxplot of the classification accuracies of the five folds using trained and nontrained Gabor-Wpsymlett dictionary . . . . . . . . . . . . . . . . . 58 


\section{Chapter 1}

\section{Introduction}

$\mathrm{T}$

HE heart is the major muscular organ of the cardiovascular system. It pumps blood into

all other organ systems of the human body and it helps deliver oxygen and other essential nutrients that are needed to maintain their physiological functions. Abnormalities in the rhythmic electromechanical contractions of the heart results in cardiac arrhythmias, when these abnormalities rise from the ventricles of the heart, they are classified as ventricular arrhythmias. Ventricular arrhythmias are serious life threatening abnormal rhythm which could be lethal and requires immediate medical attention. The need to understand the different types of ventricular arrhythmias and the differentiation between them motivates ventricular arrhythmia signal analysis approaches from an engineering perspective. In these approaches, the focus has been to develop tools to either better understand the mechanisms that cause ventricular arrhythmias or to provide an automated detection method to predict arrhythmic events by analyzing the heart's electrical activity during arrhythmias and to deliver appropriate therapy in a timely manner.

\subsection{Electrophysiology of the Cardiovascular System}

The cardiovascular system circulates blood to transport nutrients, oxygen, carbon dioxide, and hormones to the body's cells and organs. Improper and inadequate circulation can result in cell and/or organ damages. The cardiovascular system consists of the heart (which acts as a pump), blood, and blood vessels. As shown in Figure 1.1, there are four chambers of the human heart, the left and right atria and the left and right ventricles. The atria and ventricles are separated by valves, 
the right atria and right ventricle are separated by the tricuspid valve while the left atria and left ventricle is separated by the bicuspid valve. The left and right chambers are separated by a muscle wall called septum. The left atria receive oxygenated blood from the lungs and pumps it into the left ventricle to be sent to the body, while the right atria receive the de-oxygenated blood from the body and then pump it to the right ventricle and from the right ventricle the blood is sent to the lungs.

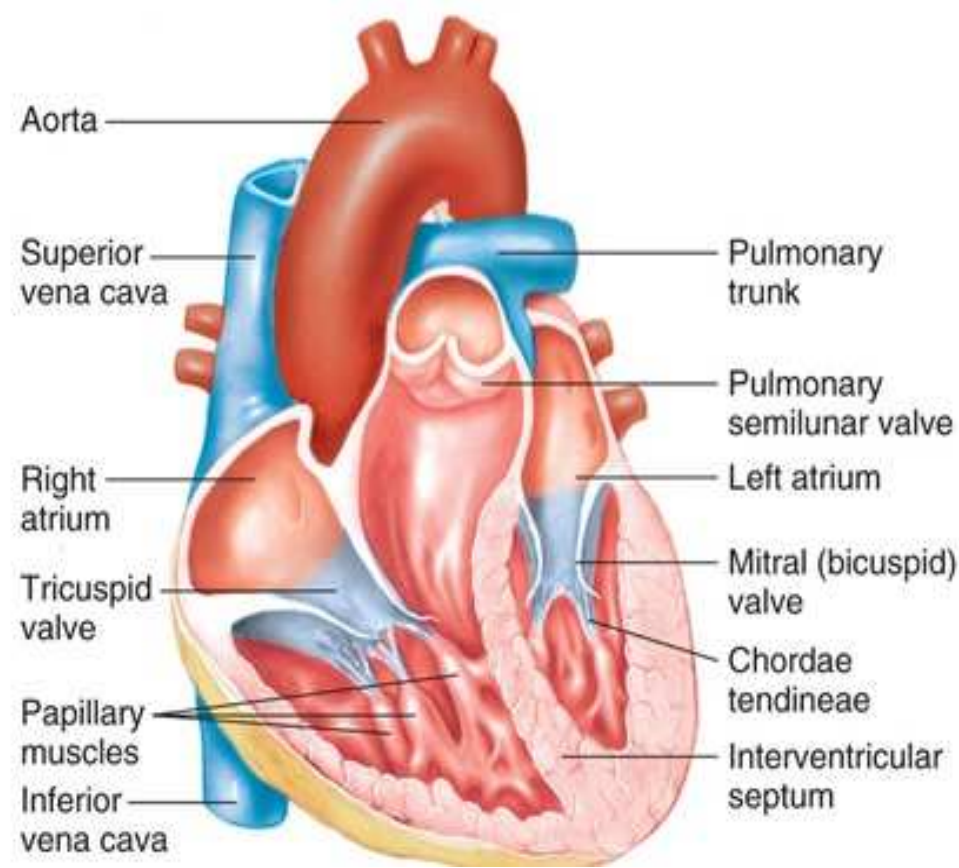

Figure 1.1: The anatomy of the heart (C)McGraw-Hill [1]

The conductive system of the heart starts from the top of right atrium to the end of the ventricles; it consists of the sinoatrial (SA) node, atrioventricular (AV) node, atrioventricular bundles, the left and right bundles of branches, and Purkinje fibres. To measure the electrical activity of the heart over a period of time, an electrical impulse is initiated at the sinoatrial (SA) node which causes the atria to depolarize. When the atria are filled with the blood that is delivered from the lungs, the pressure build up causes the atria to contract and open the bicuspid and tricuspid valves. After the electrical impulse reaches the atrioventricular (AV) node, it travels through the atrioventricular fibers (AV bundles) and then the slow conducting Purkinje fibers. The electrical excitation 
of the ventricles in addition to the pressure build up causes the semilunar valves to open and the ventricles to contract (depolarization). This electrical conduction pathway is shown in Figure 1.2. The rhythmic electrical activity of the heart can be an indication of a normal functioning cardiovascular system.

For a normal sinus rhythm such as the one that is shown in Figure 1.3, the contraction of the atria generates a P wave of the ECG signal where the electrical impulse travels from the sinoatrial (SA) node to the atrioventricular (AV) node. While the QRS complex captures the depolarization of the ventricles, and the $\mathrm{T}$ wave captures the repolarization (relaxation) of the ventricles. On average, an adult human heart beats 75 times per minute [1]

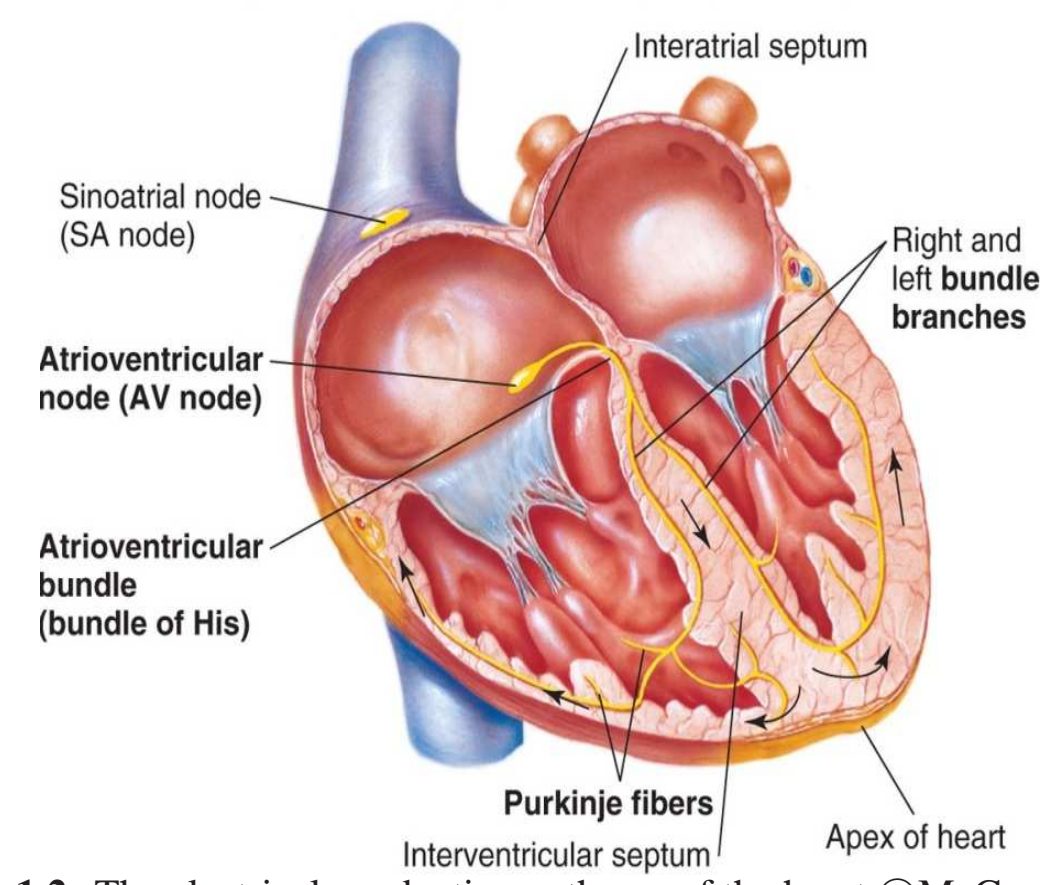

Figure 1.2: The electrical conduction pathway of the heart (c)McGraw-Hill [1]

\subsection{Ventricular Arrhythmias}

Arrhythmias that are originated from the abnormal electromechanical contractions of the heart's ventricles are called ventricular arrhythmias. Since the function of the ventricles is to pump blood out to the organs of the body, arrhythmias related to them are fatal. The two most common ventricular arrhythmias that affect peoples lives are ventricular tachycardia (VT) and ventricular fib- 

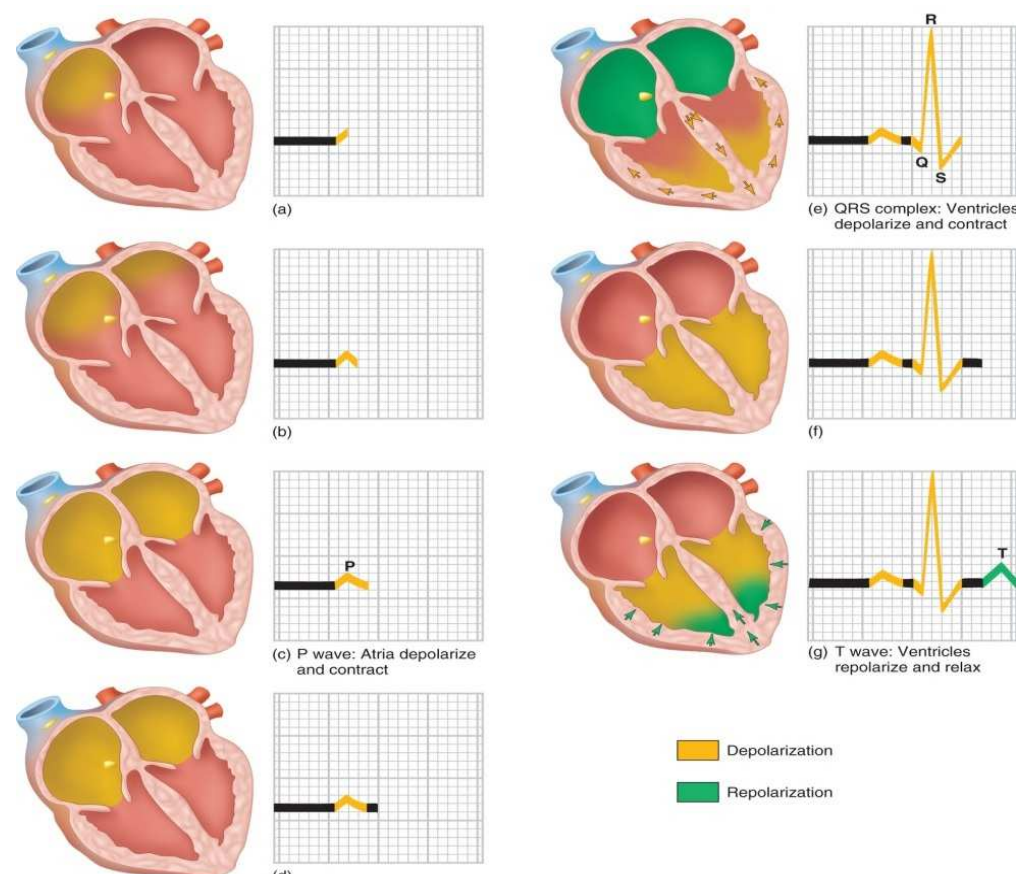

Figure 1.3: The electrical conduction of normal sinus rhythm (C)McGraw-Hill [1]

rillation (VF). In VT the heart beats rapidly (110-250 bpm) and may lead to VF if untreated [3]. In VF the heart beats extremely rapidly (more than $300 \mathrm{bpm}$ ) in an uncoordinated way which causes the heart to stop pumping blood efficiently. VF is the most dangerous arrhythmia because it may lead to sudden cardiac death (SCD) if not treated immediately. In Canada more than 40,000 SCDs occur annually [4]. An example of ECG during each type of ventricular arrhythmia is provided in Figure 1.4. Due to high mortality rates and serious impact on quality of life ventricular arrhythmias have been an area of focus in research. With the advances in newer technologies, analysis tools and treatment options are being developed to help minimizing related deaths, however with limited success.

\subsubsection{Ventricular Tachycardia}

VT is usually a predecessor to VF, it is abnormally fast but mostly organized rhythm in the ventricles. Patients with VT may experience the symptoms of angina (chest discomfort), fainting, dizziness, palpitations, and shortness of breath. These symptoms may start and stop suddenly, and in some cases there may be no symptoms [5]. Treatment options for VT vary depending 

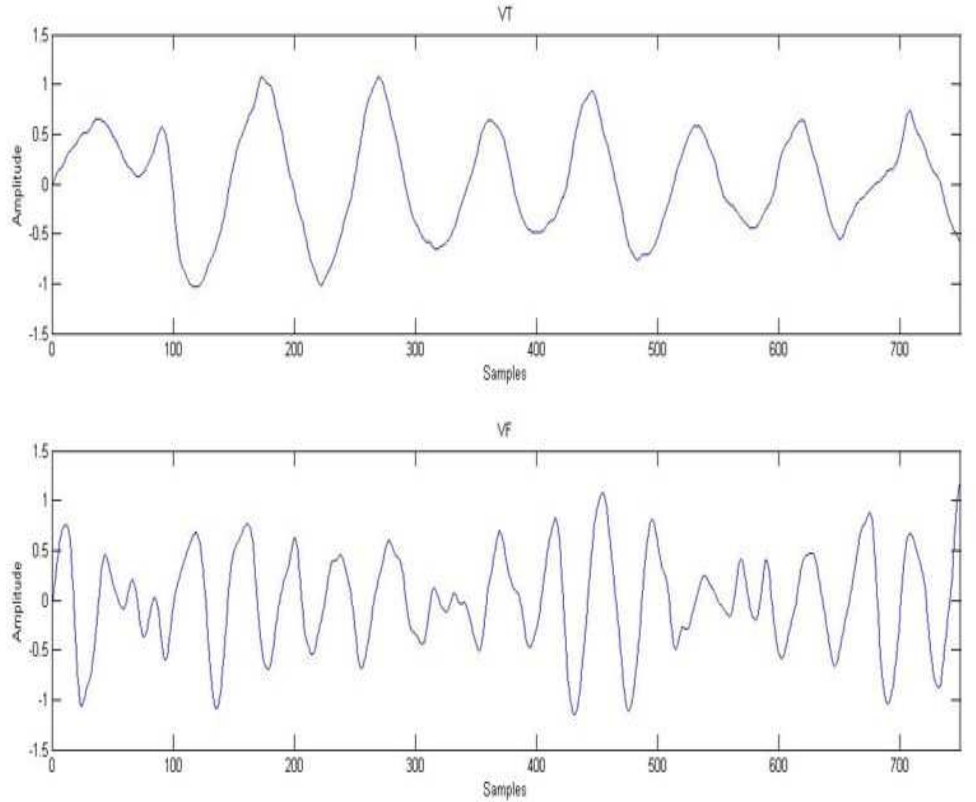

Figure 1.4: Three second ventricular tachycardia (top), three second ventricular fibrillation (bottom)

on the type and the stability of patient condition. Intravenous anti-arrhythmic medications are usually the first line therapy, electrical defibrillation and CPR might be required in critical conditions. For long-term treatment options oral anti-arrhythmic medications may be needed. Some VT may be managed by radio-frequency catheter ablation, where certain tissues that causes the irregular rhythm are ablated which will stop the abnormal rhythm [5]. There are two subclasses of VT, monomorphic ventricular tachycardia (MVT) and polymorphic ventricular tachycardia (PVT). MVT has an organized structure where all of the QRS waves will be symmetrical, at each beat the morphology of the signal is maintained, the MVT originates from a single focus within the ventricles [6]. In PVT, the QRS waves will not be symmetrical, and with variation in morphology of the arrhythmic signal [7], in PVT there are multiple ventricular foci with the resultant QRS complexes [8]. Ventricular tachycardias that last longer than 30 seconds are categorized as sustained VT, while if it is less than 30 seconds are categorized as non-sustained VT. 


\subsubsection{Ventricular Fibrillation}

VF is more lethal than VT; it is a life threatening arrhythmia. When a person suffers VF, the electrical impulses in the ventricles become arrhythmic, where many random foci sources in the ventricles along the cardiac muscle attempt to take control of the heart which causes the ventricles to have uncoordinated asynchronized contractions [9]. This will result in ineffective or no blood flow to major organs, including the brain, causing oxygen deprivation that may lead to malfunction of organs and may lead to death. The person who has a VF episode might become unconscious due the poor blood circulation to the brain and muscles, where usually cardiopulmonary resuscitation (CPR) is applied within short period of time. In addition to the CPR an electrical shock through the chest is applied in the attempt to restart the heart and restore normal heart rhythm. The shocks are not always successful in restoring normal heart rate. Patients who are at risk to develop VF require installation of implantable cardioverter defibrillator (ICD). ICDs are programmed to detect or predict abnormal heart rhythm and either perform pacing maneuvers or deliver electric shocks to restore normal rhythm. In study of sudden death in ICD recipients, 20 out of 25 recipients received appropriate ICD therapies before death, where 16 out of the 25 were tachyarryhthmia associated [10]. VF symptoms may occur within minutes to an hour before collapsing such as chest pain, dizziness, nausea, irregular rapid heartbeat, and shortness of breath [11]. When comparing the ECG of ventricular arrhythmias to the normal sinus rhythm ECG, we can see significant differences in the signal structures as shown in Figure 1.4. In the figure the VT shows regular contractions similar to normal sinus, but without the rest period between contractions and different QRS shape, while the ECG of a patient suffering from VF has irregular, unorganized and non-rhythmic signal characteristics.

\subsection{ECG Analysis During Ventricular Arrhythmias}

\subsubsection{Time-Domain Analysis}

Over the years, researchers have presented many methods to detect or classify ventricular arrhythmias. In the literature time domain features such as ECG amplitude and inter-beat R-R interval has 
been used for classifying arrhythmias $[12,13]$. Sequential hypothesis testing was used by Thakor et al. to detect VF and VT. In their proposed method the signal was compared to a threshold of generating a binary sequence, then the probability distribution of the binary sequence was used to detect arrhythmias using Walds sequential hypothesis testing procedure [14]. Another time domain analysis for the discrimination of tachycardia arrhythmias such as VT and supraventricular arrhythmias from the normal sinus rhythm was multi-way sequential hypothesis testing [15]. In this algorithm the likelihood function was calculated of the atrio ventricular delay measurements, and compared to a threshold to discriminate between the signals [15]. Threshold crossing sample count (TCSC) was also used in literature to detect VF [16], it is an improved algorithm of the threshold crossing algorithm. Throne et al. compared four different time domain techniques to discriminate tachycardia from normal sinus rhythm. The compared techniques were the bin area method (BAM), correlation waveform analysis (CWA), derivative area method (DAM), and accumulated difference of slope (ADS) [17]. Another comparison of algorithms for the application of recognition of VF was performed by Clayton et al. [18]. The compared techniques were threshold crossing intervals $(\mathrm{TCl})$, peaks in the auto-correlation function (ACF), signal content outside the mean frequency (VF filter), and signal spectrum shape $[18,19]$. These time domain algorithms are a glimpse of many different time domain techniques that exist in literature.

\subsubsection{Frequency-Domain Analysis}

Since time domain does not provide the spectral information of the analyzed signals, frequency domain analysis is used to evaluated spectral properties. When a signal is transformed into the frequency domain it is decomposed into a combination of sine and cosine functions of different frequencies. Researchers used Fourier transform to study ventricular arrhythmias from the perspective of classification and prediction. Frequency analysis has been used in literature to detect VF and VT arrhythmias, Jekova et al. [19] used VF-filter leakage algorithm for VF detection. In this algorithm a narrow band-stop filter was applied with a central frequency that is equivalent to the mean signal frequency which results in a VF-filter leakage [19]. Algorithms such as spectral band analysis have also been used in VF detection. During VF the spectral components of the signal 
results narrow band of frequencies compared to sinus normal rhythm [19]. In spectral analysis for arrhythmia analysis, the parameters such as the median, dominant, edge frequency and the amplitude of the dominant frequency have been used to predict the success of the resuscitation [20,21]. Other parameters such as the centroid frequency, peak power frequency, spectral flatness, and energy were used for the prediction outcome of the defibrillation of patients that were experiencing ventricular fibrillation [22]. Frequency analysis is good for stationary signals, however ECG signals during arrhythmias are non-stationary signals in nature, therefore, time-frequency analysis has also been used for various arrhythmic applications such as characterization and classification.

\subsubsection{Time-Frequency Domain Analysis}

In time-frequency analysis the analyzed signals are studied in both time and frequency. This type of analysis is good for time varying signals such as ECG signals during ventricular arrhythmias. In general time-frequency analysis can be divided into two categories; the time-frequency energy distribution based approaches mostly used for signal representation, and adaptive time-frequency decomposition approaches that are optimal for feature extraction and signals characterization. In the adaptive time-frequency decomposition, the signal is decomposed and approximated using different time-frequency basis functions from a dictionary $D$ that are well localized in both time and frequency domains. Due to the adaptive nature of this approach, the signal approximations can be achieved as accurately as possible depending upon the characteristics of the time-frequency dictionary used.

In literature the time-frequency analysis have been used in the field of arrhythmic signals detection and classification. Short time Fourier transform (STFT) has been used in the application of the prediction of the heart rate variability [23]. Afonso et. al [24] used the short time transform to compare the time-frequency distribution of normal sinus rhythm, ventricular tachycardia, ventricular flutter, and ventricular fibrillation in addition to the smoothed pseudo Wigner Ville distribution, and cone-shaped kernel distribution [24]. Wavelet analysis has been used to classify and characterize ventricular arrhythmias $[25,26]$. Khorrami et al. compared Continues Wavelet Trans- 
form (CWT), Discrete Wavelet Transform (DWT), and Discrete Cosine Transform (DCT) in the application of ECG arrhythmias classification using two pattern classifiers [27]. DWT coefficients were also used as features to detect and classify four different arrhythmias such as ventricular tachycardia (VT), supra ventricular tachycardia (SVT), ventricular fibrillation (VFIB) and ventricular flutter (VFL) [28]. A CWT based wavelet analysis using singular value decomposition (SVD) was proposed to classify ventricular arrhythmias in the work of Balasundaram et. al. [29]. Adaptive time-frequency decomposition based approaches have also been reported for detection and classification of arrhythmic signals; features extracted from the time-frequency basis functions of the dictionary was used to classify between arrhythmias. Depending on the application a time-frequency dictionary were used. Dictionary design has been used to separate the ventricular activity and the atrial activity of ECG signals for the purpose of characterizing and modeling atrial fibrillation [30,31], also, it has been used in heartbeat classification applications [32,33]. Recent works on dictionary learning algorithms based on K-SVD and LC-KSVD approaches has been reported for ECG beat classifications, modeling of atrial fibrillation, and ECG reconstructions and compression applications [34-37], more details of these works will be discussed in the upcoming chapter.

\subsection{Motivation}

Ventricular arrhythmias such as VF and VT have different signal morphologies and their optimal therapy options are different. As mentioned earlier VT signal usually has more organized signal structure, while the ECG of VF signal is more irregular, chaotic signal. While there are many existing works in literature that uses various methods to detect and classify ventricular arrhythmias, identifying and learning meaningful underlying signal structures in terms of adaptive time-frequency basis functions could lead to better understanding of the subclasses within these arrhythmias and especially the overlap zone between them.

The primary objective of this thesis is to learn and model the discriminative signal structures

of the VT and VF arrhythmias using a time-frequency decomposition approach. Considering the 
time-varying nature of these arrhtyhmias, modeling the discriminative signal structures using signal adaptive time-frequency dictionaries as base dictionary would be ideal. In achieving this goal, the proposed work will explore a dictionary learning algorithm (LC-KSVD) that jointly optimizes time-frequency dictionaries by minimizing both reconstructive and discriminative errors. The resulting discriminative sparse code could serve as a representative model that will lend itself as a compact descriptor for further feature analysis and characterization. The outcome of this work may lead a way to identify ventricular arrhythmia specific discriminative time-frequency space which may be useful in decision making algorithms on the choice of therapy. An initial study of this approach has been recently accepted and published [38].

The detailed block diagram of the proposed approaches in achieving the above objectives is shown in Figure 1.5. 


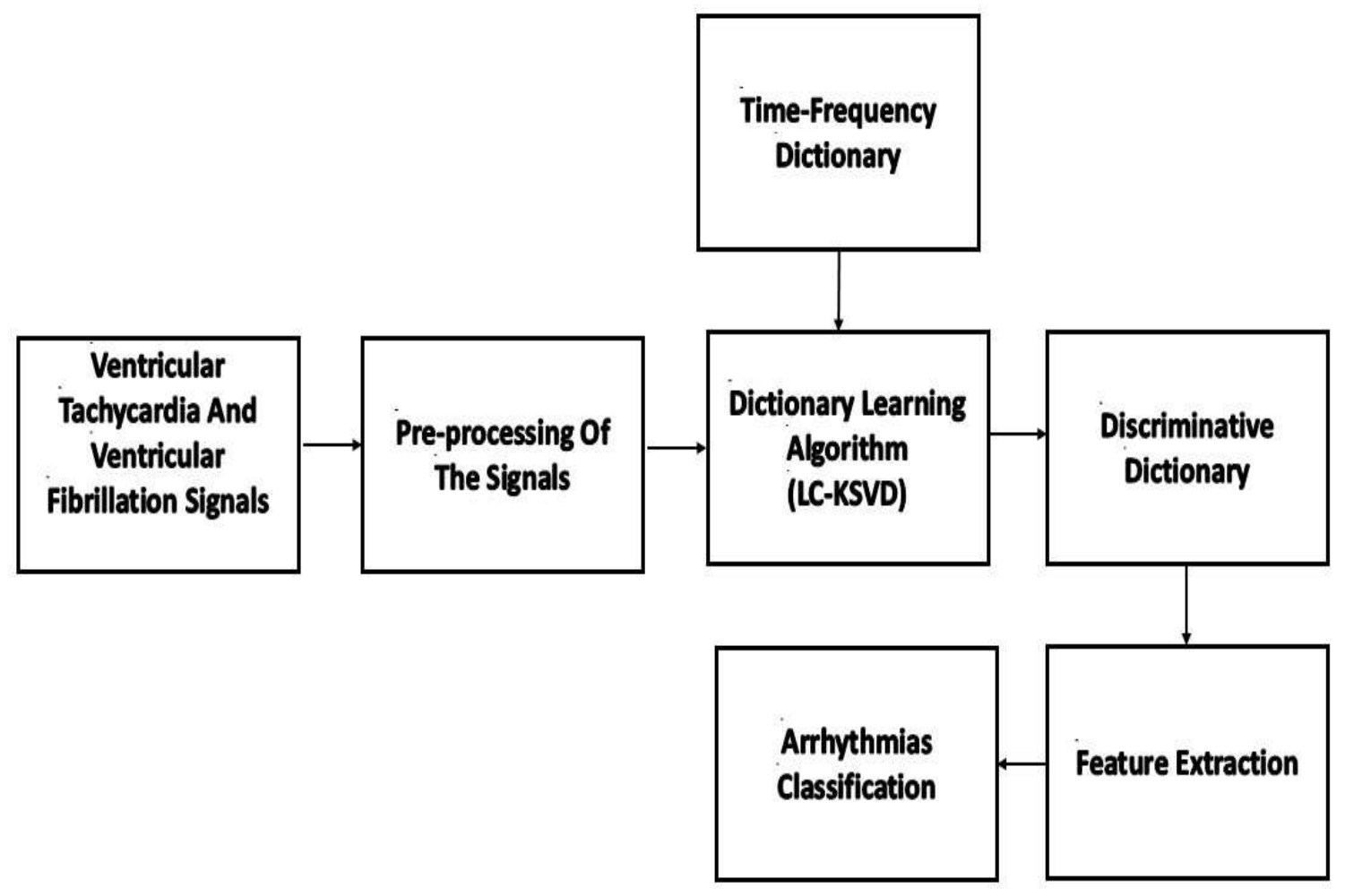

Figure 1.5: Thesis outline

\subsection{Thesis outline}

The thesis is outlined as follows:

- Chapter 2 - Methods Background. This chapter will provide information on the background of signal processing and pattern classification tools that are used in this thesis as well as specific background information on the literature survey that is related to the signal analysis methods.

- Chapter 3 - LC-KSVD Parameters for Ventricular Arrhythmia Analysis. This chapter will 
present the LC-KSVD dictionary learning process and detail on the choice of LC-KSVD parameters which are data dependent.

- Chapter 4 - Feature Extraction and Pattern Classification. This chapter will present the feature extraction and pattern classification using the discriminative sparse code representation of the two arrhtyhmias. Results for various scenarios including comparative performance evaluation are presented and summarized.

- Chapter 5 - Conclusion and Future Works. This chapter will summarize the thesis with conclusions and directions for future work, as well as the potential applications of the proposed work. 


\section{Chapter 2}

\section{Methods Background}

$\mathrm{N}$ this chapter the background information on methods used in this thesis are presented. The
chapter details different time-frequency approaches and leads to specific approaches suitable for the application at hand. In addition, the chapter also presents classification methods that will be used for performance evaluation.

\subsection{Time-Frequency Domain Analysis}

One of the early techniques of time-frequency analysis is short time Fourier transform (STFT). In STFT, the signal is divided into smaller segments where the segmented signals are assumed to be stationary. The segments are then multiplied by a window function (nonzero, usually a Hann window, Butterworth, Gaussian bell, etc). While sliding the window function along the time axis, a two-dimensional representation of the signal is computed as shown in equation 2.1 [39],

$$
Y(k, l)=\sum_{n=0}^{N-1} y(n) g(n-l) e^{\frac{-i 2 \Pi k n}{N}}
$$

where $g(n-l)$ is the discrete window function with $l$ shifts.

Short time Fourier transform gives a fixed resolution in both time and frequency determined by the fixed window function. Narrow window gives good time resolution but poor frequency 
resolution while wide window gives good frequency resolution but poor time resolution. When analyzing a VF signal it is hard to localize the occurrence of time of a particular frequency due to its non-stationary nature. Therefore, shrinking of the window $g$ provides a better localization in time for the frequencies occurring in the specific window. The drawback of making the window length shorter is that it worsens the frequency resolution which makes it hard to determine accurate energy of the frequency. Due to this limitation of STFT, a multi-resolution analysis is developed called Wavelet transform. It is similar to STFT, however the signal is multiplied by a mother wavelet (e.g morlet) and the transform is computed separately for different segments. The width of the window of the mother wavelet is adaptively changed as the transform is computed depending on the time and the frequency content of the signal [39] as shown in Figure 2.1 [2]. Mother wavelets are mathematical functions that are localized in both time and frequency domains and have specific properties. The wavelet transform implementation is given by the equation 2.2 [39],
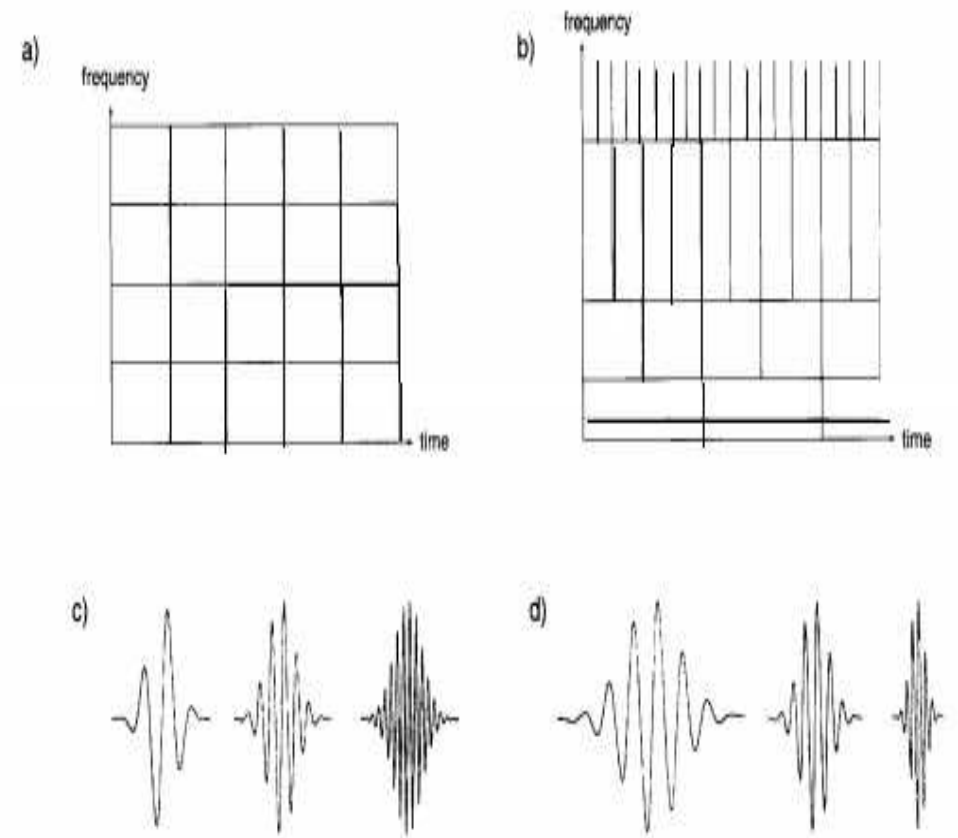

Figure 2.1: a) Coverage of the time-frequency plane for the STFT. b) Coverage of the timefrequency plane for the wavelet. c) Corresponding basis function of the STFT. d) Corresponding basis function of the wavelets [2] 


$$
y(s, l)=1 / \sqrt{s} \sum_{n=1}^{N} y(n) \psi^{*} \frac{(n-l)}{s}
$$

where $l$ represent the discretized time, $s$ the scale parameter and $y(s, l)$ represents the wavelet coefficients for the discrete time signal $y(n)$. The scaling parameter $s$ is related to the frequency, when the scale shrinks or expands the time window of the mother wavelet frequency characteristics changes accordingly. Due to having the frequency parameter being proportional to the scale of the window function, the wavelet bases will not provide a good estimate on the frequency content of waveforms that are well localized by their Fourier transform (not flexible enough) [40]. This leads to the advent of other time-frequency decomposition approaches. One such signal approximation approach is the matching pursuit [40].

Matching pursuit (MP) [40] is a greedy algorithm that expresses any signal $f$ by decomposing it into a linear expansion of waveforms from a dictionary $D$. In Hilbert space $L^{2}(R)$, the matching pursuit iteratively selects a waveform from a redundant dictionary $D=\left(g_{\gamma}(t)\right)_{\gamma \in \Gamma}$ to best approximate part of the signal. MP is a non-linear expansion but it maintains an energy conservation that will guarantee its convergence. The waveforms in the dictionary $D$ are called atoms; if $D$ is a time-frequency dictionary then the atoms are well localized in time and frequency. Time-frequency atoms can be generated by scaling $s$, translating $u$ and modulating $\xi$ the function $g(t) \epsilon L^{2}(R)$ defined by equation 2.3 [40],

$$
g_{\gamma}(t)=\frac{1}{\sqrt{s}} g\left(\frac{t-u}{s}\right) e^{i \xi t}
$$

where $\gamma$ is the index of the atoms in the dictionary.

Any signal $f(t)$ can be represented by different atoms and can be written as in equation 2.4. [40]: 


$$
f(t)=\sum_{n=-\infty}^{+\infty} a_{n} g_{n \gamma}(t)
$$

where $a_{n}$ is the expansion coefficient.

The matching pursuit adaptive decomposition starts first by finding an atom $g_{\gamma 0}$ in the redundant dictionary $D$ that best match the inner structure of the signal $f$, after the inner product is computed between the $f$ and $g_{\gamma 0}$, a residue $R f$ is computed (equation 2.5) [40]:

$$
f=<f, g_{\gamma 0}>g_{\gamma 0}+R f
$$

In this decomposition the $\left|<f, g_{\gamma 0}>\right|$ is maximized and the residual $\|R f\|$ is minimized. There after the $R f$ is sub-decomposed following the same procedure by finding an atom from the dictionary that best match the residue and then computing the inner product between the new residue and its best matching atom (equation 2.6) [40].

$$
R^{n} f=<R^{n} f, g_{\gamma n}>g_{\gamma n}+R^{n+1} f
$$

This sub-decomposition continues until the residue ideally reaches zero, a threshold is set, or a maximum number of iterations is reached. After $m$ decompositions the signal is expressed as in equation 2.7 [40],

$$
f=\sum_{n=0}^{m-1}<R^{n}, g_{\gamma n}>g_{\gamma n}+R^{m} f
$$

with an energy conservation equation $2.8[40]$ as the following:

$$
\|f\|^{2}=\sum_{n=0}^{m-1}\left|<R^{n}, g_{\gamma n}>\right|^{2}+\left\|R^{m} f\right\|^{2}
$$


A summary of the Matching Pursuit Algorithm is provided in the Algorithm 1 box below.

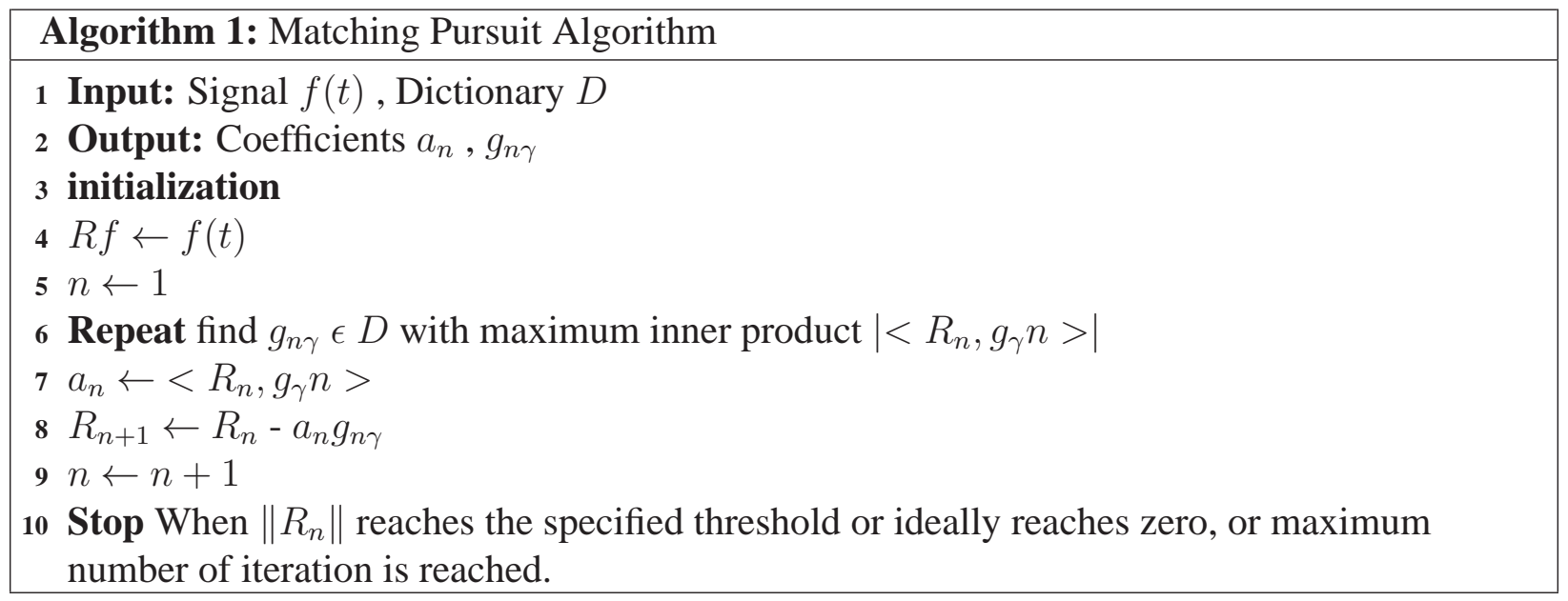

One of the enhancements of the MP algorithm is the orthogonal matching pursuit (OMP). Orthogonal matching pursuit follows the same technique as the standard matching pursuit except that it adds an extra step. When an atom is chosen to match part of the signal, it will be chosen so that the residual is always orthogonal to the atom. Hence, the same atom cannot be selected more than once [41]. In OMP, the coefficients of all the dictionary elements that are chosen are recalculated by solving the least-squares problem [41].

\subsection{Time-Frequency Dictionaries}

As mentioned earlier to perform matching pursuit decomposition, well localized time-frequency (TF) atoms are used, where they can be generated by scaling $s$, translating $u$ and modulating $\xi$ the function $g(t) \epsilon L^{2}(R)$ as shown in equation 2.3. The collection of family of TF atoms form a dictionary. Matching pursuit decomposition depends on the choice of the dictionary. There are two types of dictionaries, parametric and data driven dictionaries [42]. The choice of the dictionary is important and application dependent. 


\subsubsection{Parametric Dictionaries}

The parametric dictionary can be generated by changing the three parameters that defines an atom $(s, u, \xi)$, which gives a storage advantage where only those parameters need to be stored [42]. The most common dictionary that is used in literature and applications is Gabor dictionary, where the window function is a Gaussian window. A one real discrete Gabor atom is defined as [40],

$$
g_{\gamma, \phi}(n)=g_{s}(n-p) \cos \left(\frac{2 \pi k}{N} n+\phi\right)
$$

with the discrete parameters of atom $\gamma=\left(s, p, \frac{2 \pi k}{N}, \phi\right)$ and discrete window function $g_{s}$ defined as [40],

$$
g_{n}(n)=\frac{K_{s}}{\sqrt{s}} \sum_{p=-\infty}^{+\infty} g\left(\frac{n-p N}{s}\right)
$$

and $K_{s}$ is used to normalize $\left\|g_{\gamma}\right\|=1$

An example of a Gabor atom is given in Figure 2.2

Dictionary selection and design is the most critical step in signal decomposition with respect to classification and characterization. When a time-frequency dictionary is used for the classification of ventricular arrhythmias, the selected atoms of the dictionary needed to be representative of each arrhythmia. The information of those atoms can then be used as features to discriminate between the arrhythmias.

Hernandes et al. [30] investigated in designing a multi-component dictionary to separate atrial and ventricular activity of ECG signals to analyze and characterize atrial fibrillation. The ventricular activity of an ECG signal has much higher amplitude compared to the atrial activity which makes it hard to detect atrial arrhythmias such as atrial fibrillation. For this particular application the dictionary was built by the union of two sub-dictionaries called multi-component dictionary, 


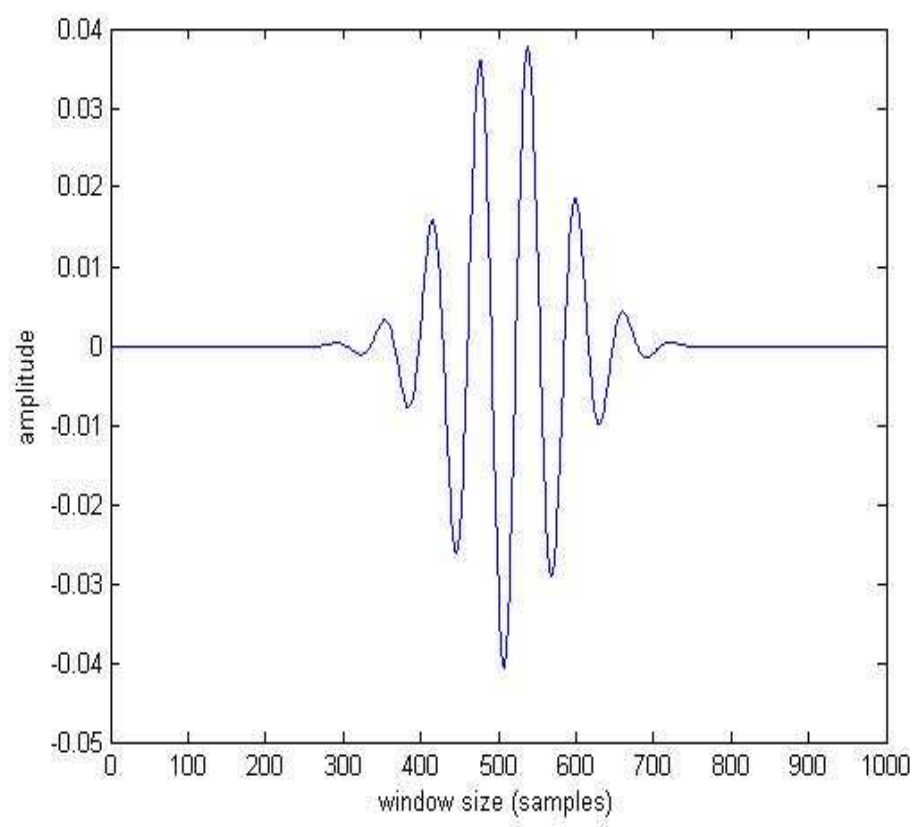

Figure 2.2: Gabor Atom

where each sub-dictionary is particularly good for describing a certain morphology of the signal. The first sub-dictionary represented the ventricular activity (VA), while the second sub-dictionary represented the atrial activity (AA). In order to have a good separation between the two activities of the signals (VA and AA) the two sub-dictionaries must have a low correlation between each other. Based on the shape and the frequency ranges of the ventricular and atrial activity, generalized Gaussian atoms were used to approximate the VA signal and Gabor to approximate the AA signal [30]. Gabor dictionary was also used in other work such as Mailh et. al [34] and Escoda et. al [31] for separation of atrial activity from the ventricular activity.

Dictionary design and selection was not only investigated in the separation of ECG signal component such as VA and AA, it was also investigated in the application of classifying arrhythmic heart beats. Christove et. al [32], performed a study to classify heart beats using the MP algorithm. They approximated each heartbeat with a small number of atomic waveforms taken from a Wavelet Packet (WP) and used its coefficients for classifications. The different types of heart beats they investigated were normal beats, premature ventricular contractions, left bundle branch blocks, right 
bundle branch blocks, and paced beats [32]. In this study they concluded that Symmlet 8 wavelet packet atom can be best correlated with the signal structures of each heartbeat class, and that 10 of such waveforms were enough to describe most of the beats in the MIT-BIH arrhythmia database with an acceptable accuracy [32]. The same dictionary was also used in other work with respect to heart beat classification [33].

\subsubsection{Learned Dictionaries}

The second type of dictionaries are the data driven dictionaries also known as learned dictionaries, these dictionaries start from an over complete redundant dictionary and get trained using a training data to adapt its content to the given signals to provide a better approximation. One of the earlier methods of dictionary learning is probabilistic approach to construct new dictionary $[43,44]$. In this approach the probability distribution of the training signals $P\left(y_{i}\right)$ is matched with the probability distribution $P(Y \mid D)$ of the input signals $y_{i}$ given a dictionary $D$. In this method the input signal y is represented as a linear combination between the dictionary elements $D$ and sparse representation $w$ with some Gaussian white noise $c$ and variance (equation 2.11) with the assumption that $P\left(y_{i} \mid\right.$ D) are independent [44].

$$
y=D w+c
$$

From equation 2.11 the prior distribution $P\left(y_{i} \mid w, D\right)$ can be defined as [44]

$$
P\left(y_{i} \mid w, D\right)=k e^{\frac{\left\|y_{i}-D w\right\|^{2}}{2 \sigma^{2}}}
$$

where $k$ is a constant. The likelihood function is computed as the following [44]

$$
P\left(y_{i} \mid D\right)=\int P\left(y_{i}, w \mid D\right) d w=\int P\left(y_{i} \mid w, D\right) \cdot P(w) d w
$$


with $P(w)=e^{\lambda}\|w\|$, because the integration is hard to evaluate the $P\left(y_{i} \mid w, D\right)$ is only calculated at the maximum values of $w$, therefore $D$ can be evaluated as [44]

$$
D=\arg _{\max }^{D} \sum_{i=1}^{N}{ }_{w i}^{\max } P\left(y_{i}, w_{i} \mid D\right)=\arg _{\min }^{D} \sum_{i=1}^{N}{ }_{w i}^{\min }\left\|y_{i}-D w\right\|^{2}+\lambda\left\|w_{i}\right\|
$$

Another dictionary learning algorithm found in literature is the method of optimal directions (MOD) [45]. The method of optimal directions is characterized by searching for the optimal atoms of a dictionary while fixing the sparse coefficients. This method follows the K-means and uses any adaptive decomposition method to update the dictionary $D$ [45]. In MOD the Dictionary $D$ is first initialized of size $N \times K$, then each training signal $y$ is approximated using vector selection algorithm as [45]

$$
\hat{y}=\sum_{i=1}^{k} w(i) d i
$$

where the $w(i)$ are the coefficients that corresponds to each atom. After the signal is approximated, the residuals for all training vectors are calculated $r=y-\hat{y}$ and the atoms are adjusted so the total MSE becomes smaller. The adjustment of the atoms is made by the following equation [45]:

$$
\tilde{d}_{i}=d_{i}+\delta_{i}, i=1, . ., k
$$

where $\delta_{i}$ denotes the adjustment of the atom. After updating the residual, the new residual for the training signal becomes [45]

$$
r=r-\sum_{i=1}^{k} w(i) \delta_{i}
$$


The update of the residual continues until the stopping criteria is reached (such as the maximum number of iterations or a constant MSE is approached) [45].

Unlike MOD where it only updates the atoms of the dictionary, in the K-SVD method [43] the dictionary is updated by updating one atom and its related sparse coefficients while all other atoms and coefficients remain unchanged. The K-SVD method is a flexible algorithm that is used in dictionary learning; it is a generalization of the K-means algorithm [43]. Like any other dictionary learning methods this algorithm is performed using an objective function (equation 2.18) which is given by [43]

$$
\min _{D, X}\|Y-D X\|_{2}^{2}
$$

In the objective function the $Y$ corresponds to the column matrix of the training signals, $D$ is a column matrix for all of the dictionary elements and $X$ is the row coefficient matrix that corresponds to the dictionary elements. K-SVD uses two stages to update, in the first stage the sparse coding is updated, where it keeps the dictionary $D$ fixed and search for coefficients of the dictionary that satisfy equation 2.18 using any type of pursuit such as OMP. The second stage of the K-SVD algorithm is updating the atoms of the dictionary $D$, in this stage the sparse matrix $X$ is assumed to be fixed and only one column of the dictionary $d_{k}$ is updated at a time. Hence, the penalty function is redefined as in equation 2.19 [43]

$$
\|Y-D X\|_{2}^{2}=\left\|Y-\sum_{j=1}^{k} d_{j} X_{T}^{j}\right\|_{2}^{2}=\left\|\left(Y-\sum_{j \neq k} d_{j} X_{T}^{j}\right)-d_{k} X_{T}^{k}\right\|_{2}^{2}=\left\|E_{k}-d_{k} X_{T}^{k}\right\|_{2}^{2}
$$

The term $E_{k}$ is the approximation error of all $y_{i}$ when the $k^{t h}$ atom is removed. In equation 2.19 the $D X$ is decomposed into a sum of $K$ rank-1 matrices, where $K-1$ atoms are fixed and the $k^{t h}$ atom is used. Finding $d_{k}$ and $X_{T}^{k}$ is done using the Singular Value Decomposition (SVD) method, this is where K-SVD gets its name from [43]. The SVD will produce the closest rank-1 matrix that 
minimizes the error. Before going through the SVD some modifications to the $E_{k}$ matrix needs to be applied to overcome the problem of having a filled $x_{T}^{k}$ matrix. First, we define [43]

$$
w_{k}\left(i \mid 1<i<K, x_{T}^{k}(i) \neq 0\right)
$$

where $w_{k}$ is the indicies that point to the signals $y_{i}$ that uses the atom $d_{k}$. Then the matrix $\Omega_{k}$ is defined of a size $N \times\left|w_{k}\right|$ that has ones on the $w_{k}(i) i^{t h}$ positions and zeros elsewhere [43]. To create a smaller matrix of $x_{R}^{k}$ we use the following equation [43], which will result in a smaller matrix with deleted zero entries:

$$
x_{R}^{k}=\Omega_{k} \times x_{T}^{k}
$$

Also multiplying the $\Omega_{k}$ with the $Y$ will create a matrix $Y_{k}^{R}$ of the signals that are using the $d_{k}$ atoms [43]. Similarly, multiplying it by the error matrix $E_{k}$ will produce the $E_{k}^{R}$ matrix. This will redefine the objective function in equation 2.19 with the minimization as the following [43]:

$$
\left\|E_{k} \Omega_{k}-d_{k} X_{T}^{k} \Omega_{k}\right\|_{2}^{2}=\left\|E_{k}^{R}-d_{k} X_{R}^{k}\right\|_{2}^{2}
$$

After this modification to the matrix, the SVD decomposes $E_{k}^{R}$ to $U \Delta V^{T}$. Then the new updated $d_{k}$ will be the first column of $U$ and the coefficient vector $x_{R}^{k}$ is the first column of $V$ multiplied by $\Delta(1,1)$ to normalize the dictionary. Then all the atoms of the dictionaries will be updated using the same method [43].

The K-SVD dictionary learning algorithm has been used in literature for ventricular cancellation and atrial modeling in the ECG of patients that are suffering from atrial fibrillation, where it models AA and cancel the VA via sparsity-base source separation approach [34]. In this approach mutual incoherent dictionaries were used (one to represent the VA and the second to represent the AA), where the signals that are sparse on one of them cannot be sparse on the other one. Similar to the dictionary design by [30] that was discussed earlier, but instead of using Gabor and generalized Gaussian dictionaries to represent AA and VA respectively, learned dictionaries were 
used. The K-SVD has been also used in literature to reconstruct ECG signals from under-sampled database on constructing a combined over-complete dictionary that consist of trained dictionary by K-SVD dictionary learning algorithm of DCT or wavelet basis dictionaries [35], having a learned dictionary achieved good approximation performance to reconstruct the data. Also the K-SVD has been used in electrocardiogram compression algorithm using over-complete dictionary [36]. A summary of the K-SVD Algorithm is provided below in the Algorithm 2 box.

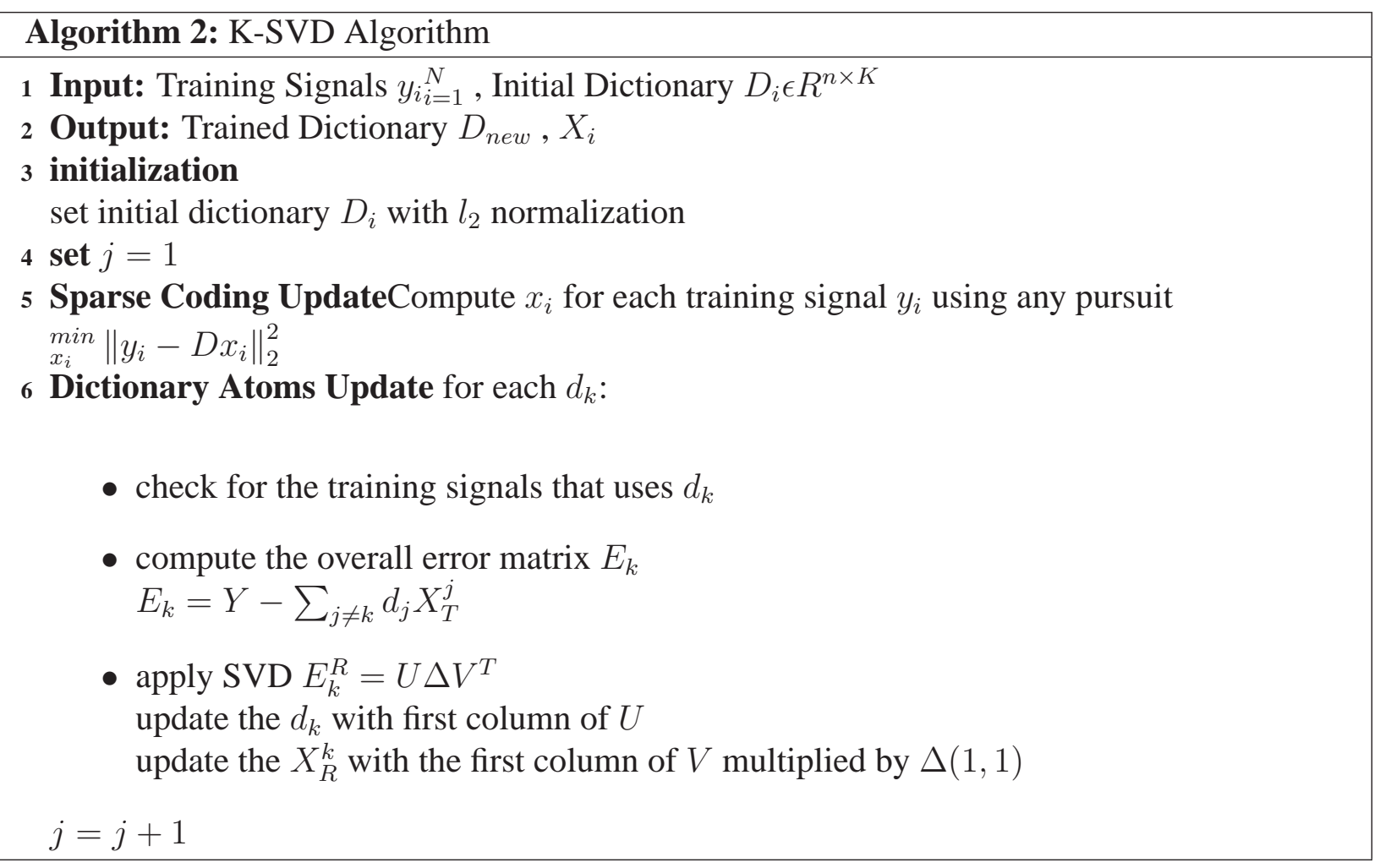

\subsubsection{Label-Consistent K-SVD}

Label-Consistent K-SVD (LC-KSVD) is an algorithm that achieve sparse coding using a learned discriminative dictionary. In the dictionary learning process, this method uses the class labels from a training data consisting of multiple groups, and associates these label information with each dictionary item to enforce discriminative sparse codes. This results in similar sparse coding for the signals that are within the same class. Furthermore, the objective function incorporates a label consistency constraint (known as "discriminative sparse-code error") and is also combined with 
the classification and reconstruction error [46], the objective function is defined as the following $[46,47]$ :

$$
<D, W, A, X>=\arg _{D, W, A, X}^{\min }\|Y-D X\|_{2}^{2}+\alpha\|Q-A X\|_{2}^{2}+\beta\|H-W X\|_{2}^{2} \text { s.t. } \forall i,\left\|x_{i}\right\| \leq T
$$

The reconstruction error is defined by the term $\|Y-D X\|_{2}^{2}$ for a set of the input signals $Y$, the dictionary $D$ and its sparse representation $X$. The second term $\|Q-A X\|_{2}^{2}$ (discriminative sparse-code error) approximates the discriminative sparse codes $Q$ using the sparse codes $X$ and the transformation matrix $A$, where $Q=\left[q_{1} \ldots q_{N}\right] \in R^{K \times N}$. The objective of the sparse code error is to provide similar sparse representations for signals of the same class, which could help improve the classification performance when using a linear classifier. The $\alpha$ parameter before the discriminative sparse-code error is used to determine the contribution of the label consistent regularization to the reconstruction. As for the third term of the objective function $\|H-W X\|_{2}^{2}$, it represents the classification error. The classifier parameters and the class labels for the input signals $Y$ are $W$ and $H$ respectively where $H=\left[h_{1} \ldots h_{N}\right] \epsilon R^{m \times N}$. The $\beta$ parameter controls the relative significance of the classification error terms to the objective function. A sparsity constraint factor $T$ was also added to ensure that each signal, when decomposed, has fewer than $T$ non-zero elements [47] [46]. As mentioned earlier in LC-KSVD the optimal solution is solved using the KSVD algorithm, therefore the equation 2.23 can be redefined as [46, 47]:

$$
<D, W, A, X>=\arg _{D, W, A, X}^{\min }\left\|\left(\begin{array}{c}
Y \\
\sqrt{\alpha} Q \\
\sqrt{\beta} H
\end{array}\right)-\left(\begin{array}{c}
D \\
\sqrt{\alpha} A \\
\sqrt{\beta} W
\end{array}\right) X\right\|_{2}^{2} \text { s.t. } \forall i,\left\|x_{i}\right\| \leq T
$$

In order to apply the LC-KSVD Algorithm an initialization of the parameters $D_{0}, A_{0}$ and $W_{0}$ is needed. To initialize $D 0$, we perform few iterations of the K-SVD for each class separate then combine the outputted dictionaries of each K-SVD. Then the label of each dictionary item $d_{k}$ is initialized based on which class it belongs. To initialize $A_{0}$, the multi-variant ridge regression is used and the solution for $A_{0}$ is computed as the following [46,47]: 


$$
A=\left(X X^{t}+\lambda_{2} I\right)^{-1} X Q^{t}
$$

similarly to the $A_{0}$ the solution for $W_{0}$ is computed as the following $[46,47]$ :

$$
W=\left(X X^{t}+\lambda_{2} I\right)^{-1} X H^{t}
$$

where $X$ is the sparse codes of the initial dictionary $D_{0}$ and training signals $Y$.

With the initialized parameters, the LC-KSVD can be performed using equation 2.23. The classification procedure of the testing signals after the dictionary learning stage using LC-KSVD is performed as follows: The trained dictionary $D_{n e w}$, transformation matrix $A$, and classifier parameters matrix $W$ are obtained, then each of the matrices are transformed using the below equations $2.27,2.28,2.29[46,47]$

$$
\begin{aligned}
& \hat{D}=\frac{d_{1}}{\left\|d_{1}\right\|_{2}}, \frac{d_{2}}{\left\|d_{2}\right\|_{2}}, \ldots, \frac{d_{k}}{\left\|d_{k}\right\|_{2}} \\
& \hat{A}=\frac{a_{1}}{\left\|d_{1}\right\|_{2}}, \frac{a_{2}}{\left\|d_{2}\right\|_{2}}, \ldots, \frac{a_{k}}{\left\|d_{k}\right\|_{2}} \\
& \hat{W}=\frac{w_{1}}{\left\|d_{1}\right\|_{2}}, \frac{w_{2}}{\left\|d_{2}\right\|_{2}}, \ldots, \frac{w_{k}}{\left\|d_{k}\right\|_{2}}
\end{aligned}
$$

When a signal $y_{i}$ is tested, its sparse representation is computed using equation $2.30[46,47]$

$$
x_{i}=\arg _{x_{i}}^{\min }\left\|y_{i}-\hat{D} x_{i}\right\|_{2}^{2}
$$

After computing the $x_{i}$, the linear predictive classifier $\hat{W}$ is used to estimate the label $z$ of the tested signal $y_{i}$ as shown in equation $2.31[46,47]$. 


$$
z_{\text {estimated }}=\arg _{z}^{\max }\left(z_{\text {actual }}=\hat{W} x_{i}\right)
$$

A summary of the LC-KSVD Algorithm is provided below in the Algorithm 3 box.

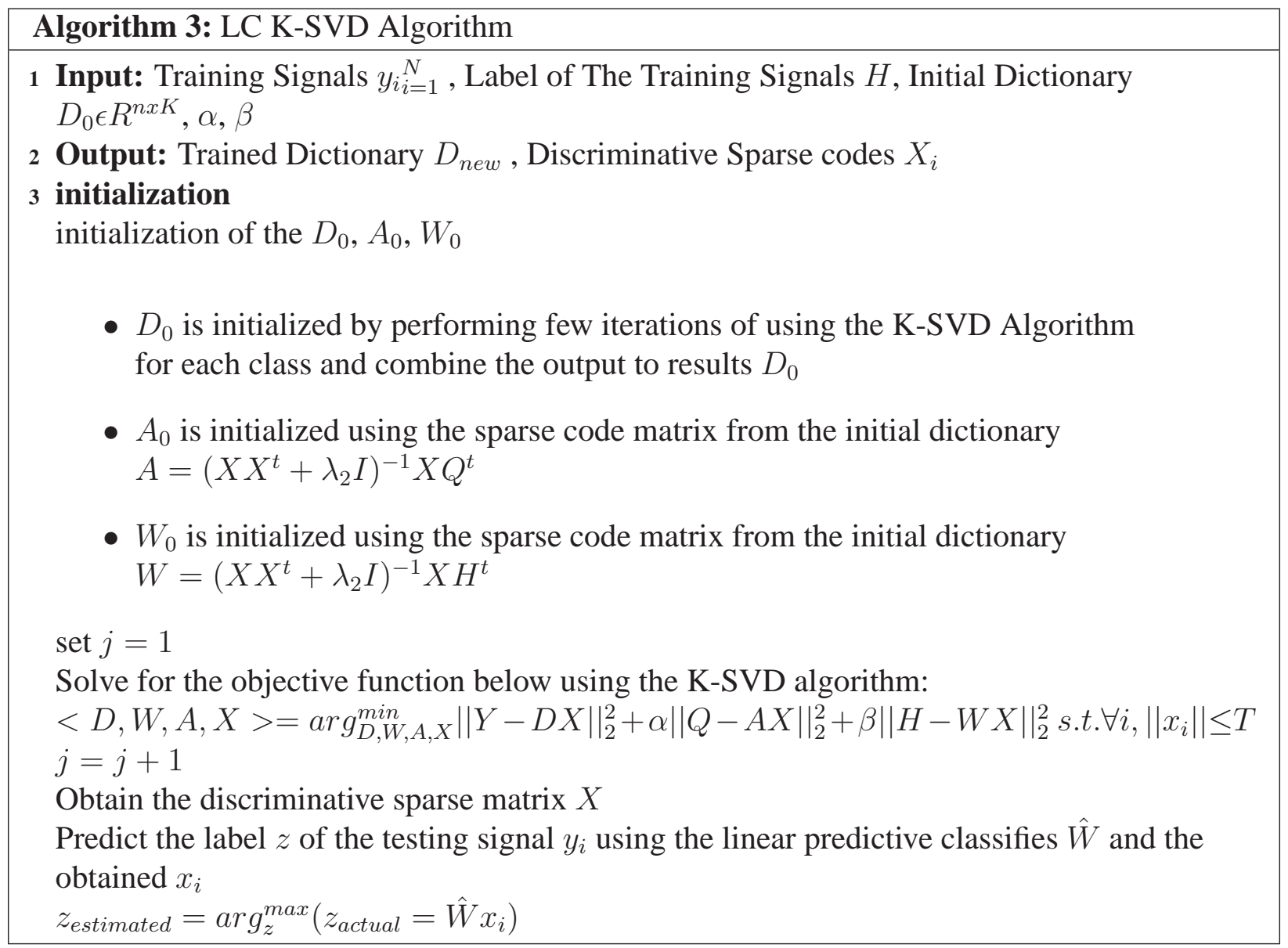

LC-KSVD has been used in literature for face, action, scene, and object category recognition [46]. From an arrhythmic perspective the LC-KSVD has been used recently in classification of electrocardiogram beats such as supra ventricular ectopic beats (SVEB) and ventricular ectopic beats (VEB) using low sampling rate [37]. The features that were used to train the dictionary were the RR interval, heartbeats interval, segmented morphology, and fixed interval morphology. It was concluded that the LC-KSVD provided enough discriminative power to classify using low sam- 
pling rate and simple features [37].

For this thesis, the LC-KSVD will be used over other approaches. Due to having different VT and VF morphologies, the sparse coding of the dictionary that represent each arrhythmia can be learned to discriminate between them. For the adaptive signal decomposition OMP will be used. The selected atoms need to be similar to the signal characteristics within the group and at the same time dissimilar between groups. Hence, selecting the proper dictionary to represent arrhythmic signal is critical. Using LC-KSVD has an advantage that it trains both the dictionary and the linear classifier simultaneously. A limitation of using the LC-KSVD to classify signals is the computational time that it takes to train a dictionary and its sparse code representation. This is a one time cost incurred in training the dictionaries. This computational complexity time is dependent on the size of the dictionary, the number of training data, and the number of iterations.

\subsection{Linear Discriminant Analysis}

After learning the discriminative sparse codes using the dictionary learning methods, we need to quantify the discriminative ability of the resulting sparse code and also would require performance evaluation over parametric choices of the dictionary learning process. In order to achieve this we need to resort to pattern classification tools. In this thesis we will be using linear discriminant analysis (LDA) based classifiers and cross validation approaches. A brief description of these approaches are presented below.

The LDA based linear classifier is built by training sets of features that are already labeled (i.e already been classified), this approach goes under the category of supervised machine learning. The number of linear functions needed for $c$ classes is $c-1$ functions, where each one will separate one class from the other classes. This will result in the feature space to be divided into different regions and each class to be assigned to one. The dimensionality in LDA is reduced by projecting the $d$ training samples $x$ onto a line or plane, a specific orientation of the line or plane can provide a good separation of the projected samples. This projection is given by the equation 2.32 [48] 


$$
y=w^{t} x
$$

where $y$ is the projection of the corresponding samples $x$ onto a line in the direction of $w$ (weight vectors). To find the best direction of $w$, we need to maximize the difference between the means $\mu_{i}$ relatively to the standard deviation $s_{i}$ of each class (scatter). For two-class classification, this criterion function is defined as [48]

$$
J(W)=\frac{\left|\tilde{\mu_{1}}-\tilde{\mu_{2}}\right|^{2}}{\tilde{s_{1}^{2}}+\tilde{s_{2}^{2}}}
$$

To express the criterion function in 2.33 as a function of $W$, we re-define the $\left|\tilde{\mu_{1}}-\tilde{\mu_{2}}\right|^{2}$ in terms of the means of the original feature space as the following [48]

$$
\left(\tilde{\mu_{1}}-\tilde{\mu_{2}}\right)^{2}=\left(\mu_{1} W^{T}-\mu_{2} W^{T}\right)^{2}=W^{t}\left(\mu_{1}-\mu_{2}\right)\left(\mu_{1}-\mu_{2}\right)^{T} W=W^{t} S_{B} W
$$

where $S_{B}$ is the between class scatter. Similarly, the summation of the variance of each class is defined as the within class scatter matrix $S_{W}$. Therefore, the criterion function 2.33 is re-defined as [48]:

$$
J(W)=\frac{W^{T} S_{B} W}{W^{T} S_{W} W}
$$

In the LC-KSVD algorithm, a multi-class linear predictive classifier is included in the objective function of LC-KSVD2 for classifier construction, while in LC-KSVD1 the classifier term is eliminated [46]. In the analysis presented in this thesis the classification accuracies will be computed using linear classifiers, also, a comparative performance evaluations of different classifiers will be presented. 


\subsection{Cross Validation}

In prediction and classifications techniques cross validation is usually needed to asses how the testing results are independent from the training data sets. It gives an insight on how the classification model will generalize to an independent data set to overcome over-fitting problems. In cross validation, the data is divided into training and testing sets. In the m-fold cross validation the data set is randomly divided into $m$ equally sized sub-samples, then one of the $m$ sub-samples will be used as a validation data for testing the model and the remaining $m-1$ sub-samples are used as training data. This is repeated $m$ times where each fold (sub-sample) is used once as the validation data as shown in Figure 2.3, and then $m$ results from the folds are averaged. In this method all observations are used for both training and validation, and each observation is used for validation exactly once. When the number of folds equals the number of observation $(m=n)$, the m-fold cross validation becomes the leave-one-out (LOO) cross validation [48].

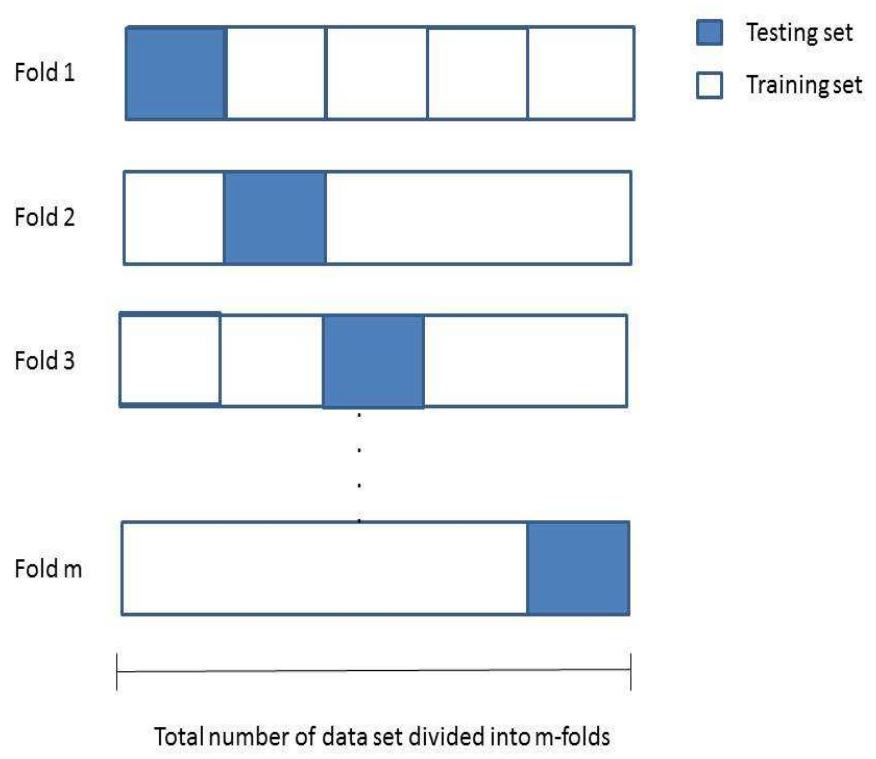

Figure 2.3: $M$ fold cross validation structure 


\subsection{Background Summary}

In this chapter the background information on relevant signal processing tools that will be used in this thesis were investigated as well as their application in literature with respect to arrhythmias. The discussed analyses were the adaptive time-frequency decompositions, time-frequency dictionaries, dictionary learning algorithms, linear discriminative analysis, and data validation. 


\section{Chapter 3}

\section{LC-KSVD Parameters For Ventricular Arrhythmia Analysis}

$\mathrm{I}^{\mathrm{N}}$

$\mathrm{N}$ this chapter the process involved in choosing optimal parameters for LC-KSVD specific to ventricular arrhythmias is presented. The chapter describes the database extraction process and signal pre-processing followed by dictionary selection. The chapter then presents the process of experimentally determining the critical parameters of the LC-KSVD for the proposed study.

\subsection{Database and Pre-processing}

The analyzed signals of this study were obtained from the MIT-BIH ventricular arrhythmia database that is publicly available through Physionet [49]. The ventricular fibrillation signals were obtained from the Creighton University Ventricular Tachy-arrhythmia Database. This database has 35 eightminute ECG recordings of patients who experienced episodes of sustained ventricular tachycardia, ventricular flutter, and ventricular fibrillation. Out of the 35 ECG recordings of this database 23 recordings had VF signals which were used to extract three second VF segments. The other database that is used is the Malignant Ventricular Ectopy Database, this database includes 22 halfhour ECG recordings of patients who experienced episodes of sustained ventricular tachycardia, ventricular flutter, and ventricular fibrillation. Out of the 22 recordings 10 contained VT episodes and were used to extract three second VT segments. In total 670 segments (335 for each arrhythmia) were extracted from the recordings and used in this thesis. The electrocardiograms from these databases were already annotated the VF and VT, therefore these annotations were used for label- 
ing the samples. Figure 3.1 illustrates one example arrhythmia signal for VF and VT respectively.

All signals were uniformly down sampled to a $250 \mathrm{~Hz}$ sampling frequency. The signals were then filtered using a bandpass filter to retain the frequency components such that low frequency artifacts and high frequency noise were removed from the ECG [50]. The cutoff frequencies used for filtering were between $0.75 \mathrm{~Hz}$ to $30 \mathrm{~Hz}$, these values were derived from the literature were ventricular arrhythmias signals were filtered between 0.3 to $30 \mathrm{~Hz}$ and 1.7 to $30 \mathrm{~Hz}$ for a comparison [21].
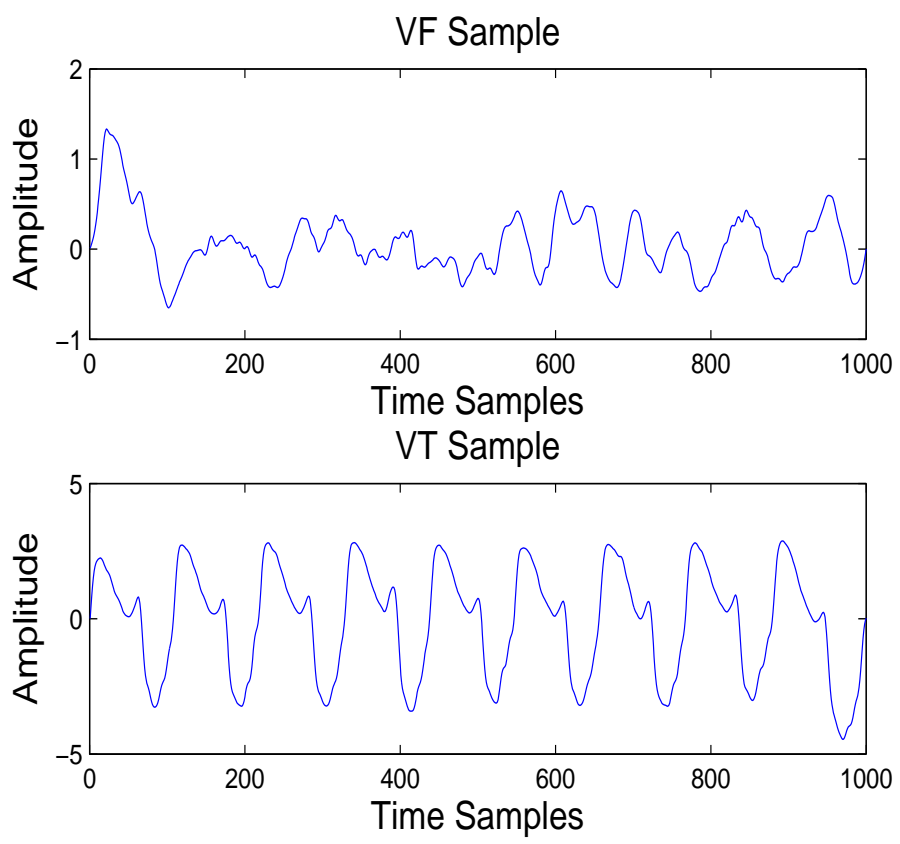

Figure 3.1: Sample signals of VF and VT

\subsection{Dictionary Selection}

The selection of the base time-frequency dictionaries used in this analysis before training were selected based on the existing works in the literature. As stated in chapter 2 of this thesis Gabor dictionary been used to approximate the atrial activity of a person with atrial fibrillation arrhythmia $[30,31,34]$. In other work a Wavelet Packet (WP) dictionary of the Symmlet mother wavelet used to describe different arrhythmic and non-arrhythmic heart beats [32,33], as well as 
the Daubechie wavelet [51]. Hence, the dictionaries that will be used in this analysis are Gabor dictionary, Symmlet wavelet, and Daubechies in least-symmetric wpsymmlet wavelet.

\subsubsection{Gabor Dictionary}

Gabor dictionary is often used in time-frequency analyses due to its optimal time and frequency energy concentration. The Gabor dictionary was constructed using the equations 2.9 and 2.10 with the discrete parameters $\gamma=\left(s, p, \frac{2 \pi k}{N}, \phi\right)$. The parameters of the constructed dictionary were selected as the follows, for the scale parameters $s=[8,16,32,64,128,512,750]$ (where $s \in 2^{m}$ where $3<m<9$ ) in addition to the window scale size of the signal. The small scale parameters capture the transient time of the signal that exist in the ventricular arrhythmias especially in VF, while the large window scale can account for the overall structure of the signals. The scale parameter is related to the time with respect to the sampling frequency, for example a window scale of 64 accounts for 0.256 seconds of the signal. For the frequency parameter the selected ranges were $f_{n}=[0-0.1]$ where $\left(f_{n}=\frac{f}{f_{s}}\right.$, the $f_{n}$ is the normalized frequency and $f_{s}$ is the sampling frequency). This range was chosen because in literature it was reported that the frequency spectrum of individual QRS complex is found in the range of 0-20 Hz [52] which corresponds to 0-0.08 of normalized frequency. The dominant frequency of ventricular tachycardia (VT) is at $4 \mathrm{~Hz}$ and the the amplitude of the spectral analysis decreases as the frequency increases $[52,53]$. Also it was reported that the frequency ranges of ventricular fibrillation (VF) are concentrated between 4-7 $H z[52,54]$.

For the time parameter $p=[0,64,128,375,512,750]$ ranges were selected with phase $\phi=[0]$ for this application. The time parameter only controls where the center of the atom is placed. Due to K-SVD limitation of having the number of the dictionary atoms lower than the number of the training signals, only specific time locations were selected instead of all of the time locations of the signals. The phase parameter specifies the offset of the cosine factor of the Gabor function where it can be any real numbers between -180 and 180 . The values 0 and 180 correspond to symmetric functions, while -90 and 90 correspond to antisymmetric functions. Using the previously 
discussed parameters a Gabor dictionary was generated with a combination of these parameters of the size $750 \times 462$. Figure 3.2 provides four different Gabor atoms that are generated in the Gabor dictionary.
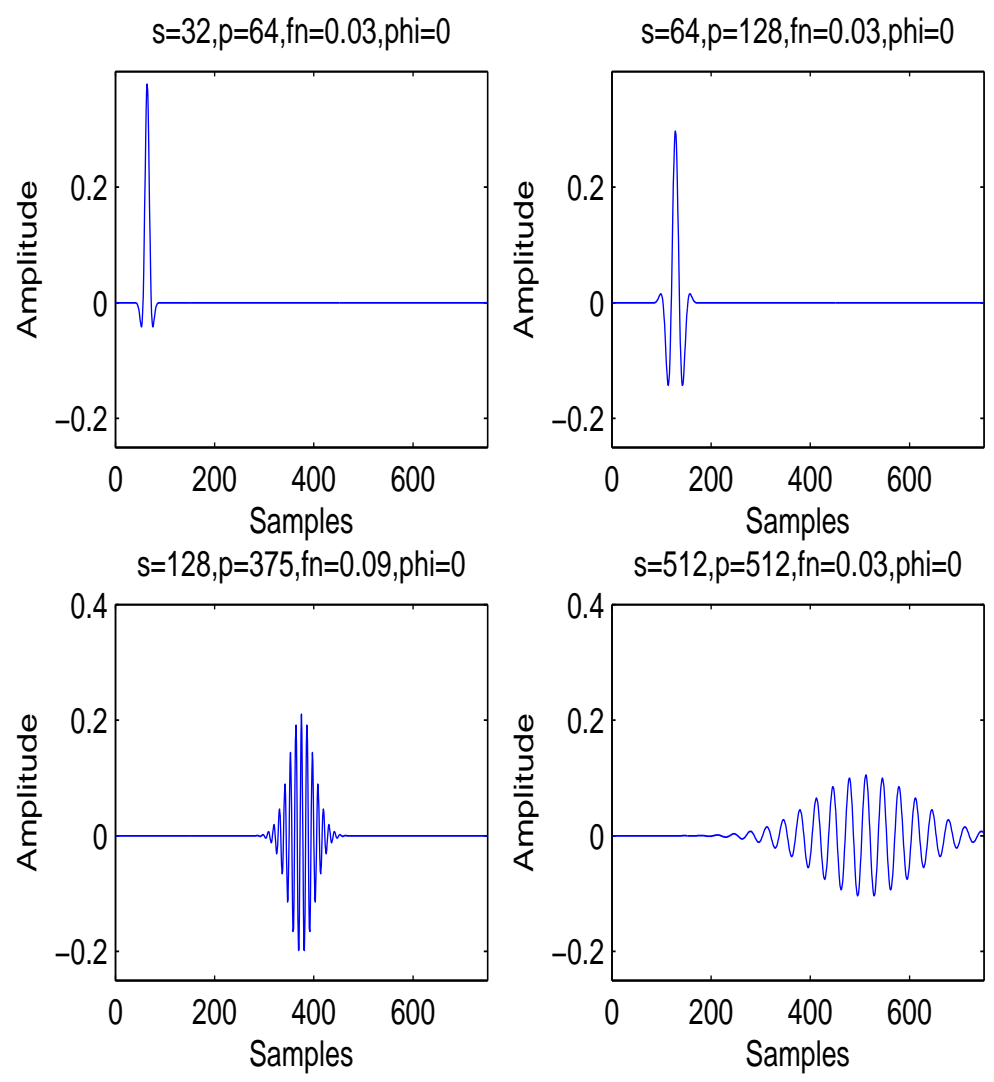

Figure 3.2: Four different Gabor atoms from the generated dictionary of different translation and scales

\subsubsection{Wavelet Dictionaries}

The Wavelet dictionaries that were used in this analysis were generated dictionaries using the three-indexed family of analyzing functions 3.1 [55].

$$
W_{j, n, k}(x)=2^{\frac{-j}{2}} W_{2}\left(2^{-j} x-k\right)
$$


where $k$ is the time-localization parameter, $j$ the scale parameter of $n^{t h}$ order [55].

The Symmlet wavelets are near symmetric, orthogonal, and biorthogonal wavelets. Their general characteristics that they are compactly supported wavelets with least asymmetry and highest number of vanishing moments, it's associated scaling filters are near linear-phase filters [56] [55]. In literature the Symlet4 wavelet has been used to represent and classify arrhythmias $[57,58]$. The wavelets used in this analysis are the Sym 4 at level 5 and Wpsym 4 Daubechies least-asymmetric wavelet packet at level 3 due to their structure that is similar to the ECG signal morphology and its properties. The generated dictionaries were of the size $750 \times 752$ each. Figure 3.3 provides four different wavelet atoms that were generated in the wavelet dictionary.
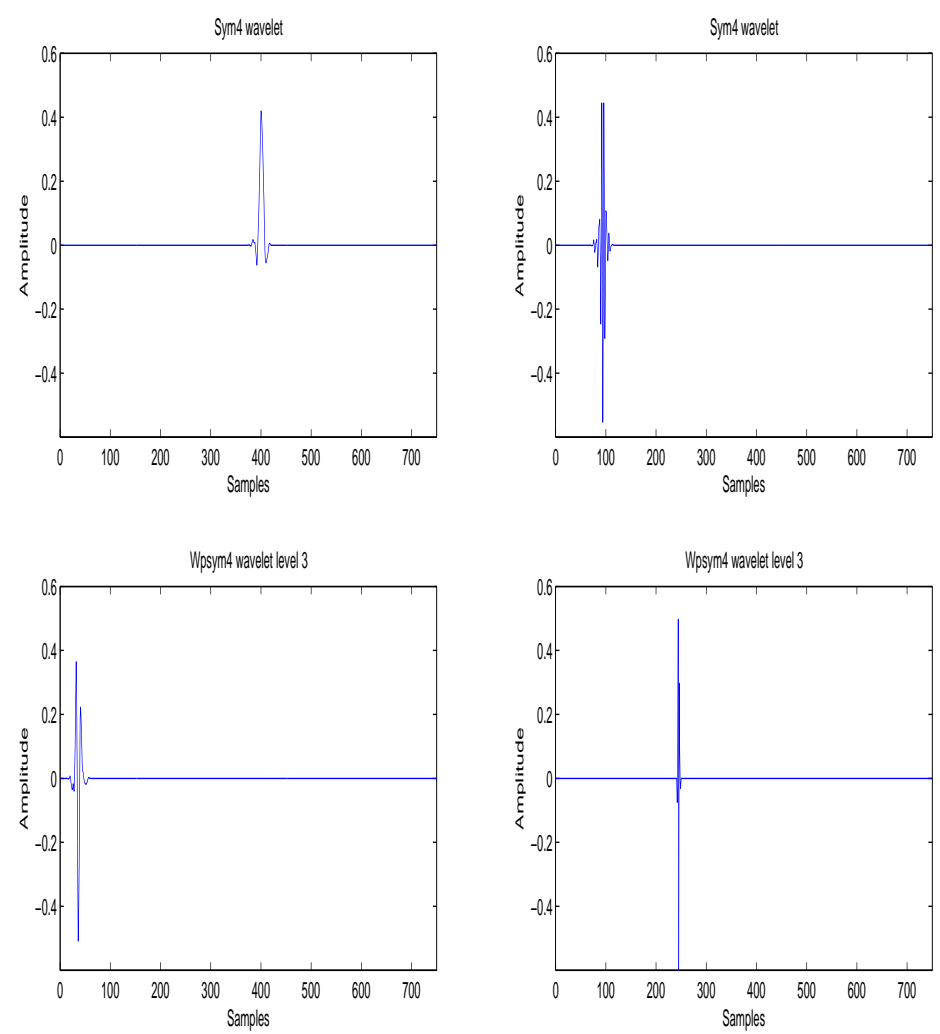

Figure 3.3: Four different Wavelet atoms from the generated dictionaries 


\subsubsection{Optimal Dictionary}

To apply the dictionary learning algorithm LC-KSVD, the number of the training signals of each arrhythmic class has to be larger than the dictionary that will be learned, this is an implementation constraint of the K-SVD algorithm [43]. Therefore, a subset of the generated dictionaries will be used for the analysis so the dictionary size is smaller than the trained signals. Hence, the chosen dictionary size was set to 230 so it can be smaller than the 268 training data sets. Before selecting the 230 atoms three different hybrid dictionaries were generated from a combination of two of the three dictionaries that were discussed previously (Dictionary 1: Gabor and Sym4, Dictionary 2: Gabor and Wpsym4, Dictionary 3: Sym4 and Wpsym4). To choose the 230 atoms of each hybrid dictionary, matching pursuit algorithm was applied on 50 random arrhythmic signals of the database, and then the first 230 atoms of the first few iterations that best describes the signals were selected to create the new optimal dictionaries for the analysis. In order to test if the new sub-dictionaries can describe other arrhythmic signals, matching pursuit was applied on different signals (not the 50 that were randomly selected) where more than $90 \%$ of the energy were captured. The following graphs demonstrate three testing signals (the original and the reconstructed) as well as the captured energy.

\subsection{Choice of Optimal Parameters}

Recalling from chapter 2 the LC-KSVD objective function is:

$$
<D, W, A, X>=\arg _{D, W, A, X}^{\min }\|Y-D X\|_{2}^{2}+\alpha\|Q-A X\|_{2}^{2}+\beta\|H-W X\|_{2}^{2} \text { s.t. } \forall i,\left\|x_{i}\right\| \leq T
$$

The $\alpha$ and the $\beta$ parameters are used to determine the contribution of the label consistent regularization to the reconstruction error and the relative significance of the classification error of the objective function respectively. $\alpha$ controls the contribution of the discriminative error term in the minimization whereas $\beta$ controls the contribution of classifier error term. These parameters are data dependent and for this analysis they were chosen based on many trials. Considering the factors from the literature [46], the optimal values of $\alpha$ and $\beta$ were searched in the range of 0.01

to 6. A subset of database (536 arrhythmia segments) was used to identify the optimal $\alpha$ and $\beta$ 

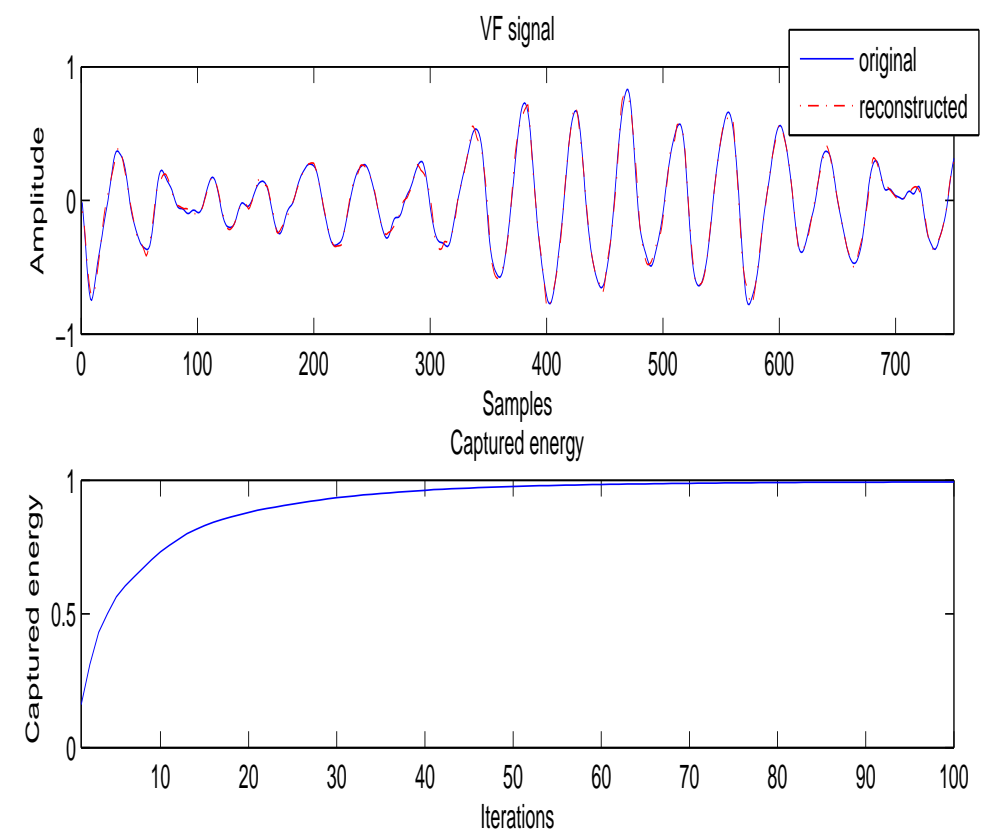

Figure 3.4: Matching pursuit on VF signal (Top) and it's captured energy (bottom) using Dictionary 1
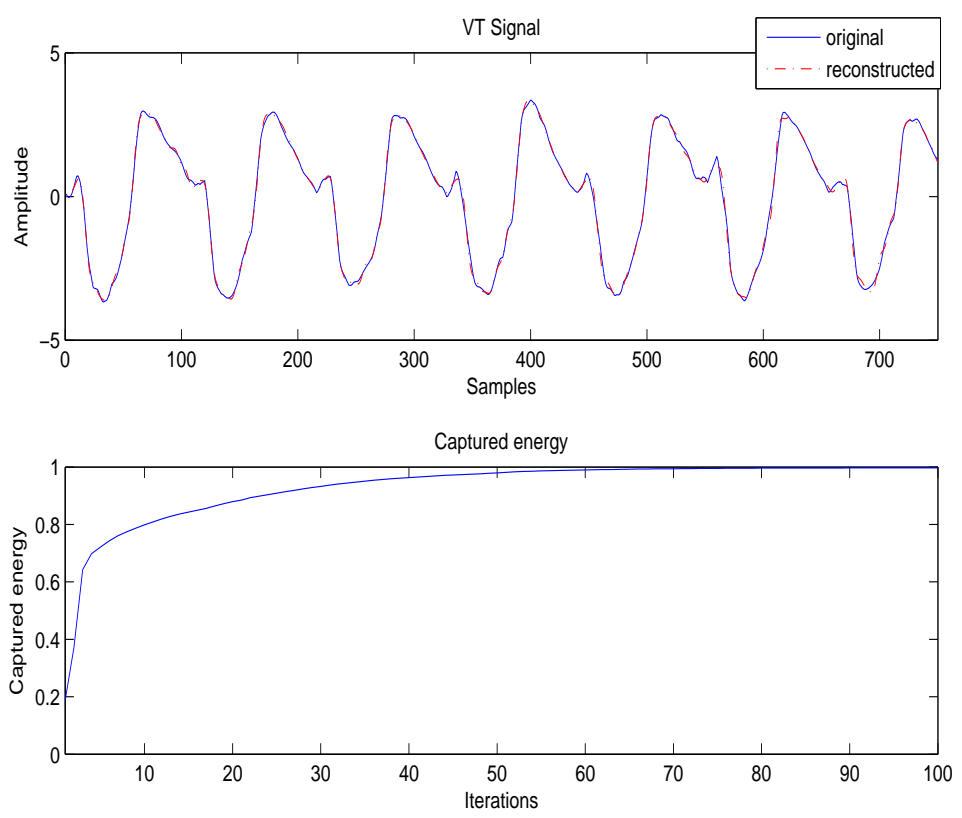

Figure 3.5: Matching pursuit on VT signal (Top) and it's captured energy (bottom) using Dictionary 2 

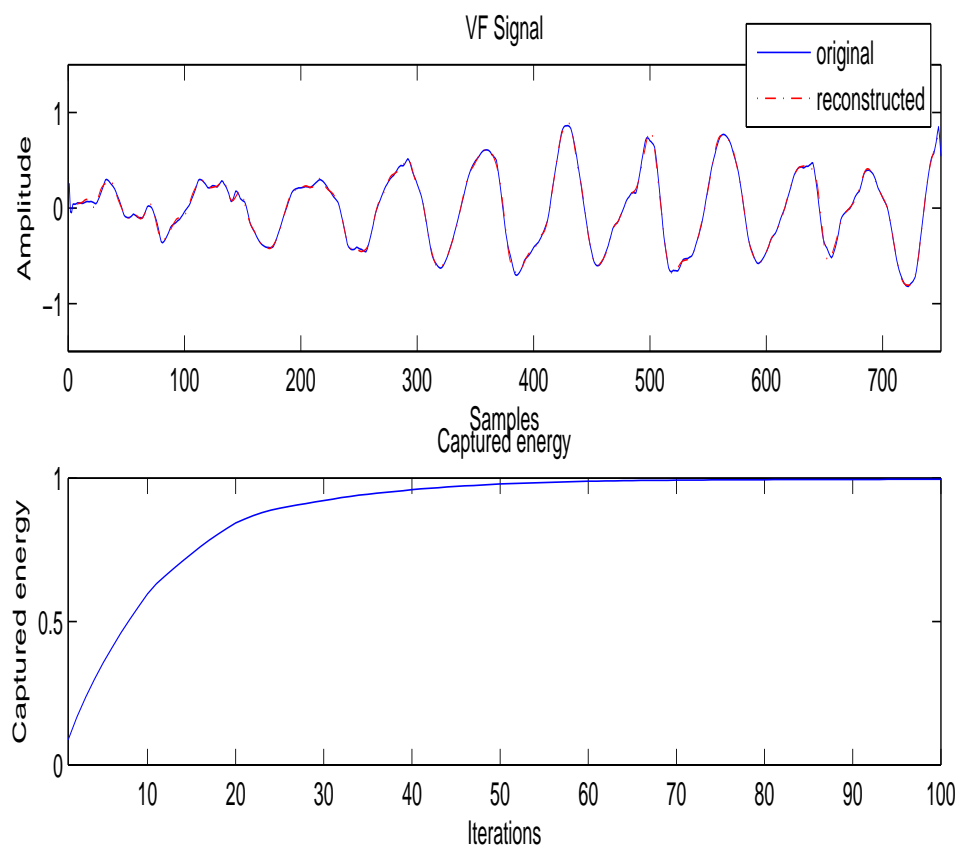

Figure 3.6: Matching pursuit on VF signal (Top) and it's captured energy (bottom) using Dictionary 3

parameters using Gabor and Sym4 dictionaries, where the choice of the dictionary and signals were at random.

First, the classification error of the objective function in equation 3.2 is eliminated (i.e setting $\beta$ to 0$)$. After 2000 iterations, the total error consisting of the reconstruction error and the discriminative sparse code error (error 1) was obtained as shown in Table 3.1, also in this table the classification accuracy of testing signals using the trained dictionary was obtained. Then the classifier error term is included in the objective function (error 2), and the total error is obtained after 2000 iterations using a combination of different $\alpha$ and $\beta$ values, as shown in Figure 3.10, as well as the classification accuracy using the different ranges (Figure 3.9).

From Table 3.1 the total error for using different $\alpha$ values ranges from 0.017 to 0.023 . Having a value of $\alpha$ less than 2 produced an error less than 1\%, while having an $\alpha$ value of 4 and 6 produced an error greater than 1\%. Having an error lower than $1 \%$ produced a higher classification accuracy compared to errors greater than 1\%. The Figures 3.7 and 3.8 show the classification accuracies for the subset of data and the error 1 respectively, as could be observed the highest classification 
Table 3.1: Classification accuracy and reconstruction error for different $\alpha$ parameters for 2000 iterations of LC-KSVD

\begin{tabular}{|c|c|c|}
\hline$\alpha$ & Classification accuracy (\%) & Reconstruction error \\
\hline 0.01 & $64.2 \%$ & 0.004 \\
\hline 0.1 & $67.2 \%$ & 0.004 \\
\hline 0.5 & $69.4 \%$ & 0.002 \\
\hline 1 & $76.1 \%$ & 0.005 \\
\hline $\mathbf{2}$ & $\mathbf{7 8 . 4 \%}$ & $\mathbf{0 . 0 0 8}$ \\
\hline 4 & $60.4 \%$ & 0.017 \\
\hline 6 & $61.2 \%$ & 0.023 \\
\hline
\end{tabular}

occurs at an $\alpha$ value of 2 with an acceptable error of less than 1\%, hence we chose the optimal value of $\alpha$ to be 2 using Gabor and Sym4 dictionary. This value was also tested using different subset of data and an optimized dictionary that consists of a combination of all three dictionaries, which produced similar results (highest classification accuracy at $\alpha=2$ ).

Similarly, when the $\beta$ parameter was included, the total error 2 and the classification accuracies were obtained. Figures 3.9 and 3.10 show the range of classification accuracies and the ranges of error 2 for different $\alpha$ and $\beta$ values ranged from $68-76 \%$ respectively. The box plots show the variations in the results for different $\beta$ values for specific $\alpha$ values. As could be observed the median of the classification range of the box plot is highest for an $\alpha$ value of 2 (with error still less than $1 \%$ ) and for the optimal value of $\beta$, the average classification accuracy using different values for $\beta$ at $\alpha=2$ was taken into consideration and arrived at an optimal value of 0.01 . The values of the errors and classification accuracies using different $\alpha$ and $\beta$ can be found in Appendix B. When the same analysis was done using different subsets of signals and the three combined dictionaries, the value of $\alpha$ being 2 and $\beta$ being 0.01 resulted in a higher classification accuracy with lower total error compared to other values. Therefore, the chosen optimal values that were used in the further analysis were $\alpha=2$ and $\beta=0.01$. As for the sparsity factor $T$, different values were pre-selected such as $(10,30,50,100)$ and tested. In the literature [46] the selected sparsity factor was 30 for their database. Using the pre-selected values for the analysis, it was concluded that higher error and lower classification accuracy were obtained by having a low sparsity factor. The higher the sparsity factor the higher the computational time it took to evaluate the sparse matrix of the signals. 
Hence, the optimal value that was selected to be used in this analysis was 50, where for any value higher than 50 there was no significant change in the error and it was merely computationally more expensive without much gain.

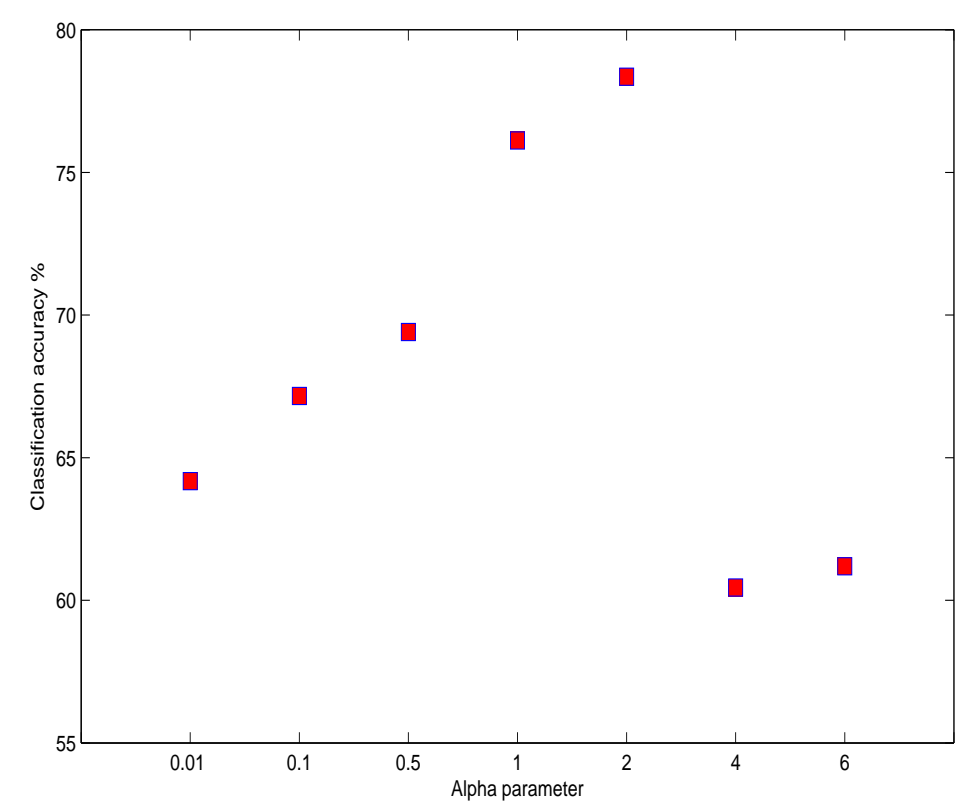

Figure 3.7: Classification accuracies using different $\alpha$ values

\subsection{Chapter 3 Summary}

This chapter discussed the choice of initial dictionaries that will be trained using the Label Consistent K-SVD algorithm. Also in this chapter reduced size of dictionaries were selected for the analysis due to the restriction of having a number of the training signals smaller than the dictionary elements. The optimal selection was then based on applying the adaptive decomposition matching pursuit on a subset of signals, and the first 230 atoms of the decomposition were used to create the energy optimal dictionaries. The chapter also presented the process involved in the selection of the parameters $\alpha$ and $\beta$ that controls the relative contribution of error in the LC-KSVD objective function stated in equation 3.2. 


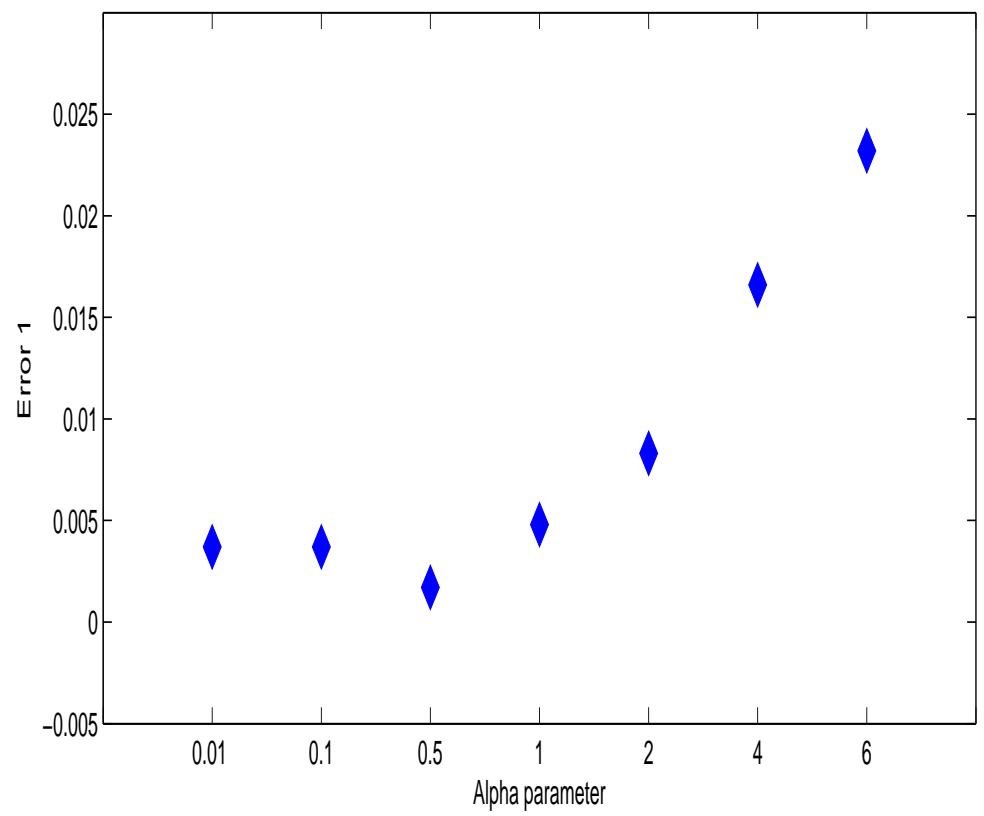

Figure 3.8: Total error 1 using different $\alpha$ values

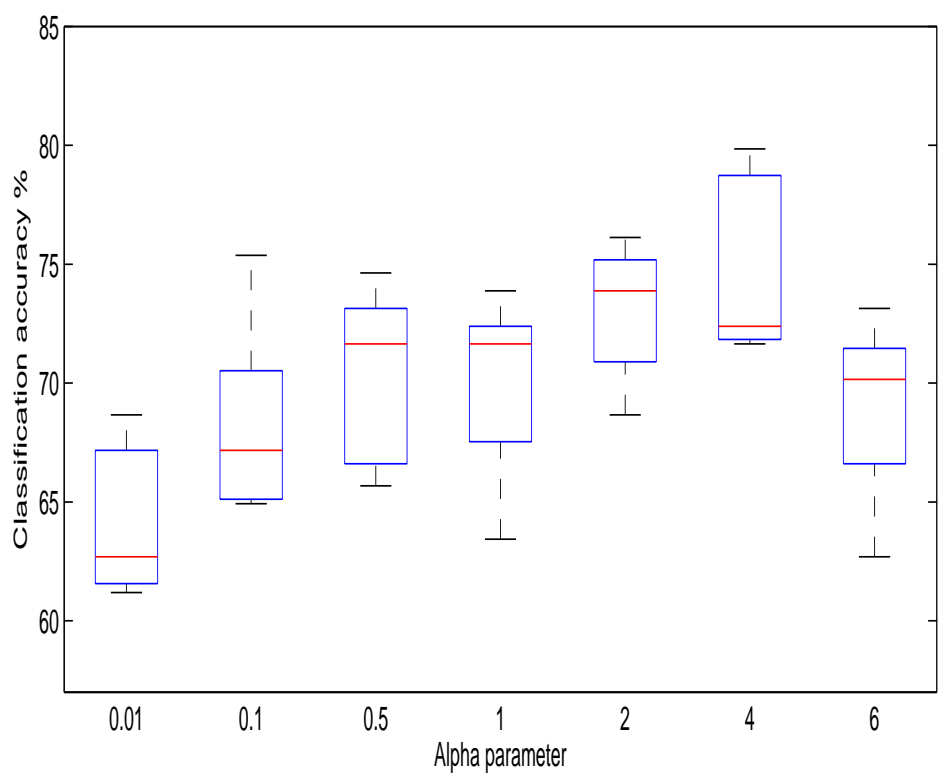

Figure 3.9: Boxplot of the classification accuracy ranges using different $\alpha$ and $\beta$ values 


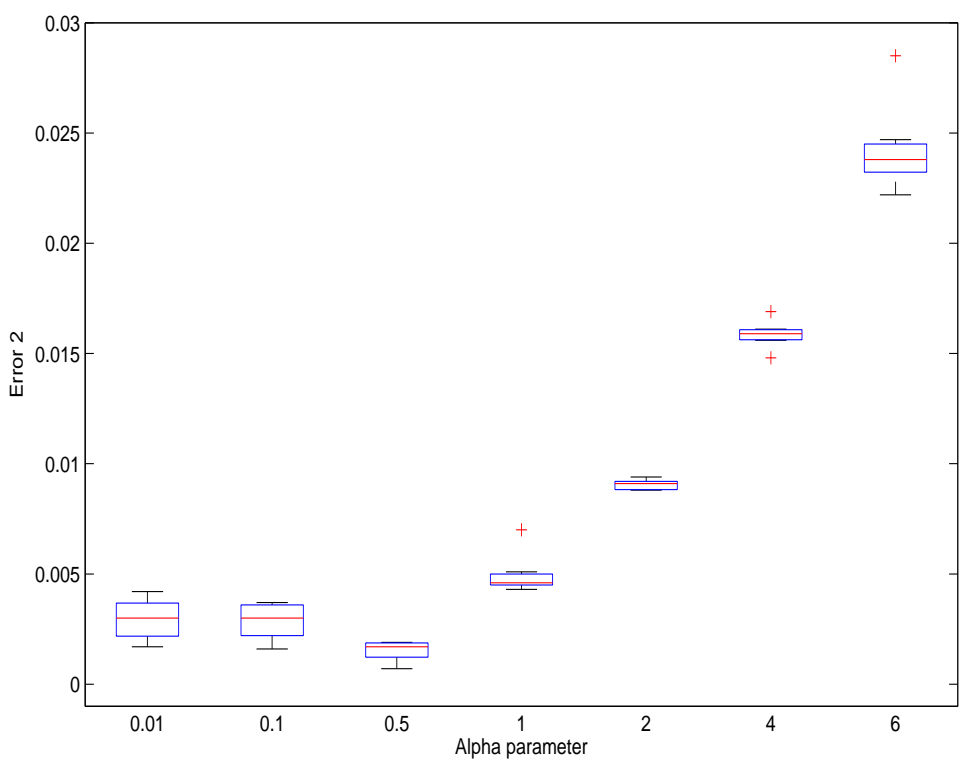

Figure 3.10: Boxplot of the total error 2 ranges using different $\alpha$ and $\beta$ values 


\section{Chapter 4}

\section{Feature Extraction and Pattern Classification}

$\mathrm{F}$

OR any classification application patterns/features are needed to be extracted in order to discriminate between classes in reduced dimensional space. Depending on the patterns distribution in the feature space the classifier type can vary between linear, quadratic or if the data distribution is complex a multi layer neural network classifier is usually used. In this chapter we present the validation of the discriminative sparse codes obtained using LC-KSVD approach by how well these codes could discriminate VT and VF. The classification accuracies were computed using linear classifiers. Comparative performance evaluations on various scenarios are also presented.

\subsection{Features/ Feature Space}

In pattern recognition techniques usually a measurable quantity is used for recognition, this quantity is called a feature. Features measure specific properties of data that is informative and discriminative. When more than one feature is used a feature vector $x$ is created, where the vector $x$ is in a $d$-dimensional space $R^{d}$ called the feature space. The feature space dimension depends on the number of feature that will be used [48]. In this analysis the feature that will be used for discriminating between the arrhythmic groups is the discriminative sparse code of the learned dictionary using LC-KSVD algorithm. For each tested data the sparse code using the learned dictionary is obtained and then multiplied by the learned classifier parameters which will results in a score estimate. The 
resultant score is a 2 row matrix, the index of the maximum score represents the classified class. Figure 4.1 represents the feature space of the score estimate of VF and VT testing signals.

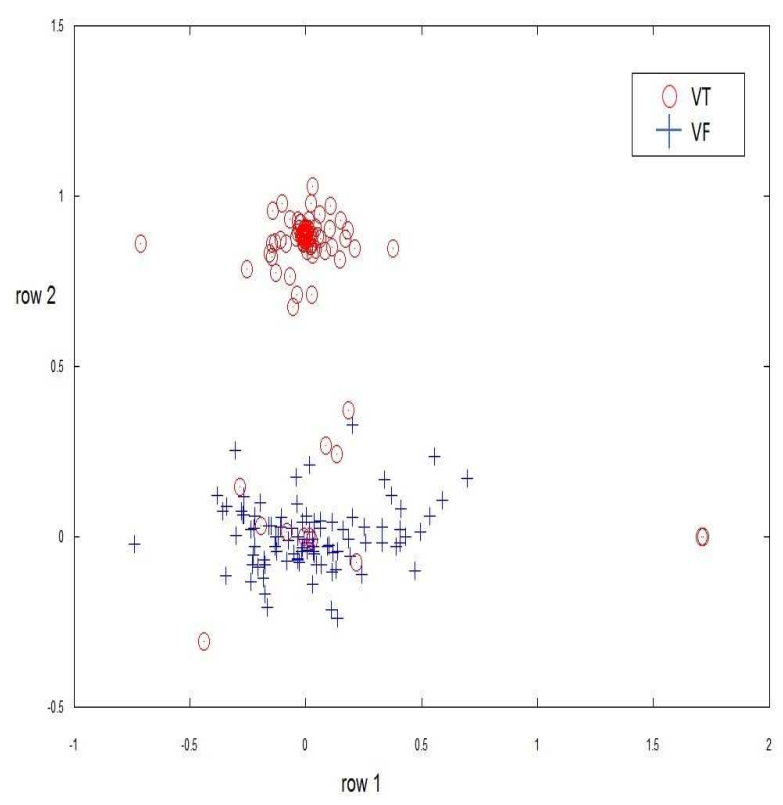

Figure 4.1: An example of feature space of the estimated score feature, where blue pluses represents VF tested signals and red circles represents VT tested signals

\subsection{LC-KSVD analysis on Arrhythmic Signals}

In this section the results are divided into two parts, analysis using the optimized dictionaries that were discussed in the previous chapter and analysis using full dictionaries (i.e., not optimized) for comparative performance evaluation. In the optimized dictionaries analysis section, the classification accuracies of the three optimized dictionaries were compared. In addition a comparison between the LC-KSVD1 $(\beta=0)$ and LC-KSVD2, where in the LC-KSVD1 the classifier error term is eliminated from the objective function and in the LC-KSVD2 it is included. In this chapter whenever it is not specified if it is LC-KSVD1 or LC-KSVD2 (i.e LC-KSVD only) it means LC-KSVD2 (the classifier error is included in the objective function). The accuracy of detecting arrhythmic signals were compared using a trained versus non-trained dictionaries, and the effect of the dictionary 
size on the classification accuracy and computational time was also discussed.

In the second part of the results analysis where the full dictionaries were used, the dictionaries were trained using an artificial increased database size by adding noise to the actual data. Classification accuracies of the three dictionaries were compared, in addition to the results of using a trained dictionary versus a non-trained one. A flow chart of the results distribution for various scenarios is illustrated in figure 4.2 .

\subsubsection{Results Using Optimized Dictionaries}

In this analysis, the $335 \mathrm{VF}$ signal segments and $335 \mathrm{VT}$ signal segments were filtered and then used for the analysis. In this analysis a 5-fold cross validation will be used to validate the results over the LOO cross validation as LOO is computationally expensive for the application in hand. The 670 signals were divided into five equal folds, where four folds used for training the dictionaries and one fold was used for testing. This was repeated five times with different validating fold at a time and the overall classification accuracy was computed as the average accuracy of the five folds.

Initially, three types of dictionaries were pre-selected for the analysis as mentioned earlier; Gabor, Symlet4, and Wpsymlet4 dictionaries. The dictionary parameters were restricted to a subset size that has been optimized in Chapter 3 to keep the dictionary size smaller than the training data set [43]. The weight factors $\alpha$ and $\beta$ were set to 2 and 0.01 respectively, and the sparsity factor $T$ to be 50. Using these parameter values, the training was done for 2000 iterations (where more than $90 \%$ of the signal energy was captured), and the classification of the testing signals was performed as described in the Chapter 2 using equation 2.31. Table 4.1 shows the different classification accuracies of VF and VT signals with different combinations of the dictionaries Gabor, Symlet4, and Wpsymlet4 wavelet. Highest overall average classification accuracy of $73.3 \%$ was obtained for the hybrid dictionary of Symlet4-Wpsymlet 4 compared to $71.6 \%$ and $72.8 \%$ for the Gabor-Symlet4 and Gabor-Wpsymlet4 respectively. The confusion matrix of the classification of the tested signals provided in Table 4.2 , where $70.1 \%$ of VF signals were accurately characterized as VF and $82.1 \%$ were accurately characterized as VT. As stated in table 4.3 the range of correctly 


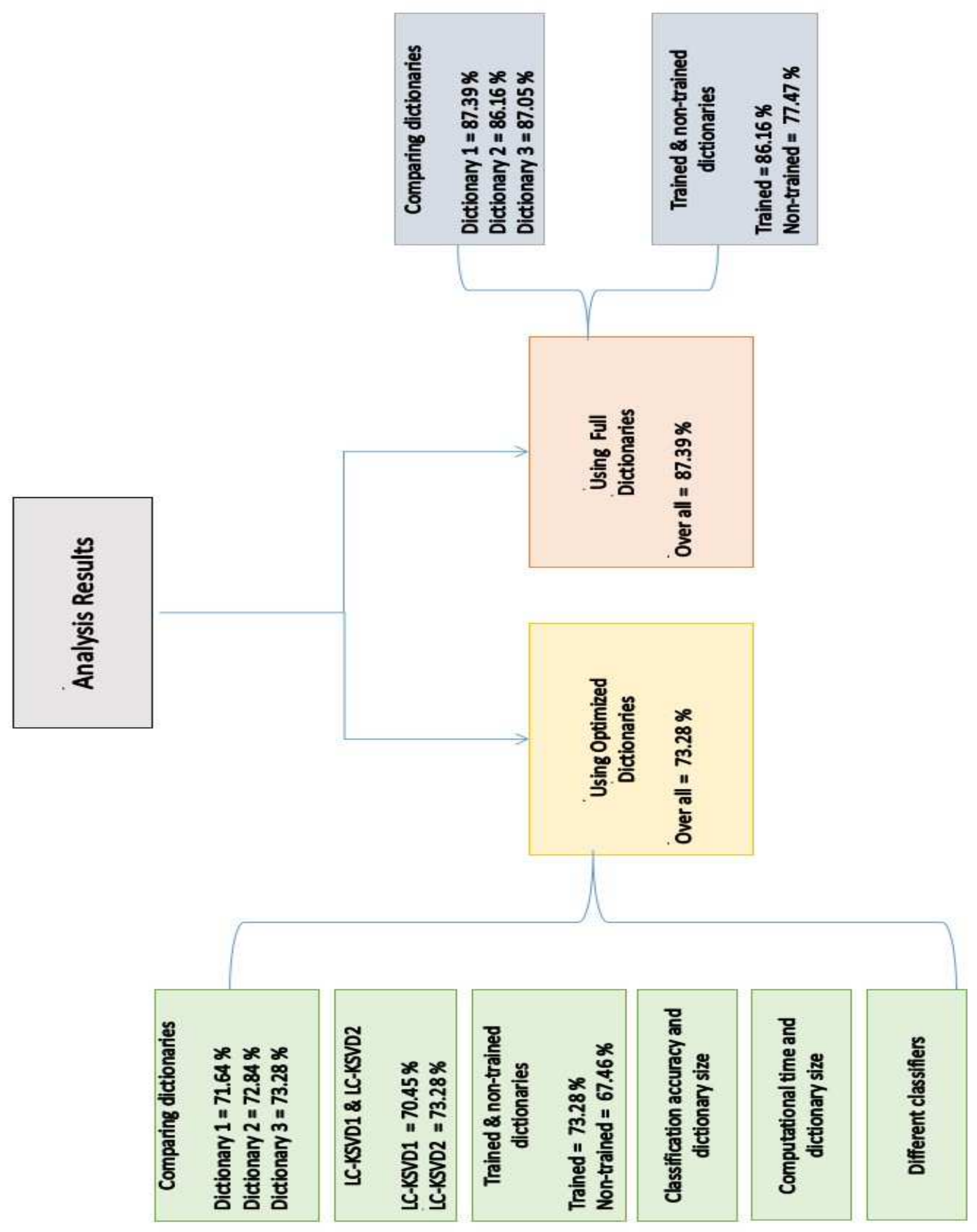

Figure 4.2: Flow chart of the results analysis 
characterizing VF and VT ranges between 52-71\% and 82-95\% respectively for different folds. The misclassified VF signals had a similar signal structure to VT signals, while the misclassified VT signals were more disorganized and had a similar structure towards VF when observed. The choice of having an optimized hybrid dictionary over individual one for training was that it performed better in classifying the arrhythmic signals compared to individual ones, while choosing a hybrid optimized dictionary of a combination of the three dictionaries did not effect the results significantly.

Table 4.1: Average classification accuracy for different combinations of the optimized dictionaries for 2000 iterations of LC-KSVD

\begin{tabular}{|c|c|}
\hline Dictionary type combination & Classification accuracy (\%) \\
\hline Gabor-Symlet4 & $71.6 \%$ \\
\hline Gabor-Wpsymlet4 & $72.8 \%$ \\
\hline Symlet4-Wpsymlet4 & $\mathbf{7 3 . 3 \%}$ \\
\hline
\end{tabular}

Table 4.2: Confusion matrix of discriminating VF and VT signals using optimized Symlet4-Wpsym4 dictionaries of LC-KSVD

\begin{tabular}{|c|c|c|c|c|}
\hline & & VF & VT & Total \\
\hline tested signals & VF & 47 & 20 & 67 \\
\hline & VT & 12 & 55 & 67 \\
\hline$\%$ & VF & $\mathbf{7 0 . 1}$ & 29.8 & 100 \\
\hline & VT & 17.9 & $\mathbf{8 2 . 1}$ & 100 \\
\hline
\end{tabular}

When the $\beta$ parameter was set to zero, which means that the classifier error was removed from the objective function, the overall classification accuracy of LC-KSVD1 using Symlet4-Wpsymlet4 became $70.4 \%$ compared to $73.3 \%$ when the classification error term was included (i.e., LCKSVD2). Figure 4.3 shows the distribution of classification accuracies of the five folds using LC-KSVD1 and LC-KSVD2 algorithms. For LC-KSVD1, a separate linear classifier was trained, 
Table 4.3: Range of the 5-folds confusion matrix of discriminating VF and VT signals using optimized Symlet4-Wpsym4 dictionaries of LC-KSVD

\begin{tabular}{|c|c|}
\hline & Classification accuracy range \\
\hline VF & $52-71 \%$ \\
\hline VT & $82-95 \%$ \\
\hline
\end{tabular}

therefore, its performance in classifying depends on how discriminative is the sparse code $x$. Depending on the fold that is used for training the dictionary, the accuracy to classify arrhythmias by including the classifier error term in the objective function or eliminating it differs. From Table 4.4, it can be shown that the five folds have lower classification accuracies using the LC-KSVD1 over the LC-KSVD2. This means that the learned dictionary sparse code $x$ using these trained folds is less discriminate when the linear classifier error is not included compared to when it is included in the objective function. For this particular example training with the classifier error term improves the ability to accurately classify arrhythmias.

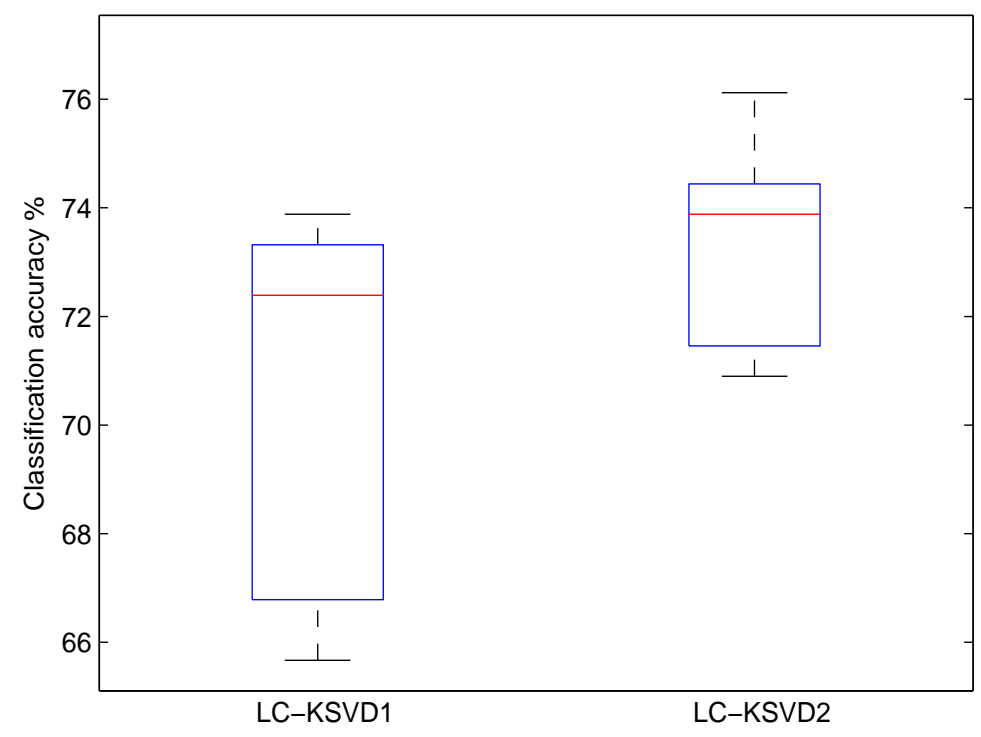

Figure 4.3: Boxplot of classification accuracies of LC-KSVD1 and LC-KSVD2 
Table 4.4: The Classification accuracy for the five folds data sets using LC-KSVD1 and LCKSVD2

\begin{tabular}{|c|c|c|}
\hline Data Sets & LC-KSVD1 & LC-KSVD2 \\
\hline fold 1 & $72.4 \%$ & $73.9 \%$ \\
\hline fold 2 & $67.2 \%$ & $70.9 \%$ \\
\hline fold 3 & $73.1 \%$ & $73.9 \%$ \\
\hline fold 4 & $73.9 \%$ & $76.2 \%$ \\
\hline fold 5 & $65.7 \%$ & $71.6 \%$ \\
\hline overall & $70.4 \%$ & $73.3 \%$ \\
\hline
\end{tabular}

The classification accuracy using the LC-KSVD trained Symlet4-Wpsymlet4 dictionary sparse code as a feature for classification was compared to the classification of the signals using the sparse code of non-trained dictionary, and as observed in Figure 4.4, the overall classification accuracy using the non-trained dictionary was observed to be less with $67.5 \%$ compared to the $73.3 \%$ for the trained dictionary. This improvement due to having more adaptive trained atoms as shown in Figure 4.5 compared to the non-trained ones in Figure 3.3. Also the Figures 4.6 and 4.7 shows the classification accuracies using trained and non-trained dictionaries combinations of Gabor-Wpsym4 and Gabor-Sym4 respectively.

Table 4.5: The Classification accuracy for the five folds data sets using the optimized trained and non-trained Symlet4-Wpsym4 dictionaries

\begin{tabular}{|c|c|c|}
\hline Data Sets & Trained Dictionary (\%) & Untrained Dictionary (\%) \\
\hline fold 1 & $73.9 \%$ & $66.4 \%$ \\
\hline fold 2 & $70.9 \%$ & $67.2 \%$ \\
\hline fold 3 & $73.9 \%$ & $67.9 \%$ \\
\hline fold 4 & $76.1 \%$ & $69.4 \%$ \\
\hline fold 5 & $71.6 \%$ & $66.4 \%$ \\
\hline overall & $73.3 \%$ & $67.5 \%$ \\
\hline
\end{tabular}

The discrimination of the arrhythmic signals using the LC-KSVD algorithm does not only de- 


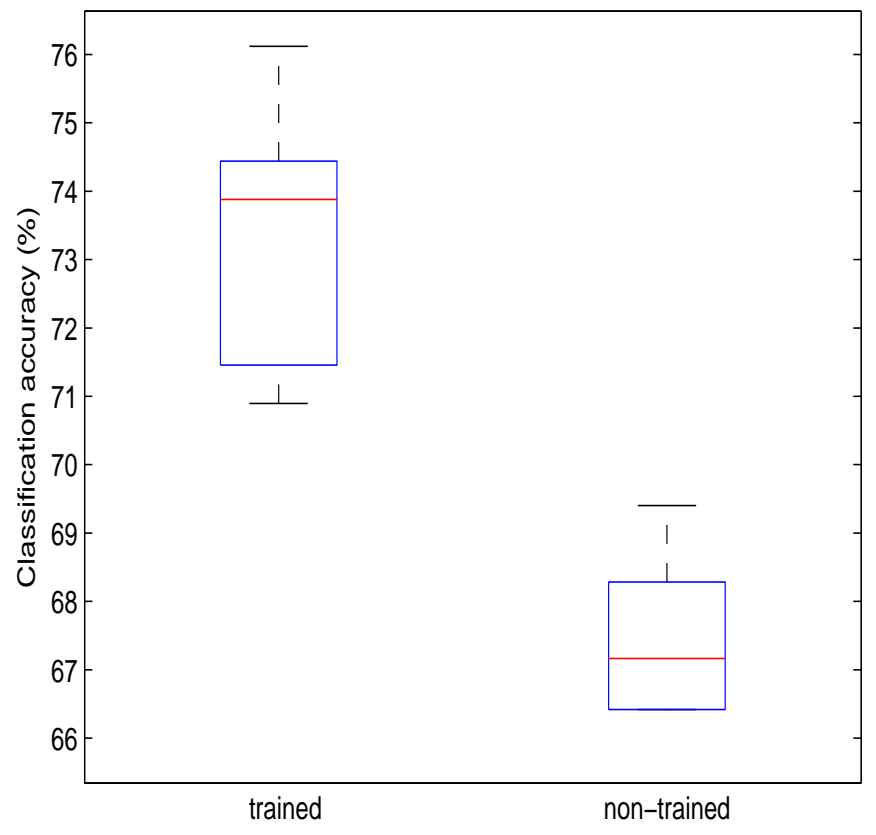

Figure 4.4: Boxplot of the classification accuracy of the five fold data sets of VF and VT using optimized trained and non-trained Symlet4-Wpsymlet4 dictionaries
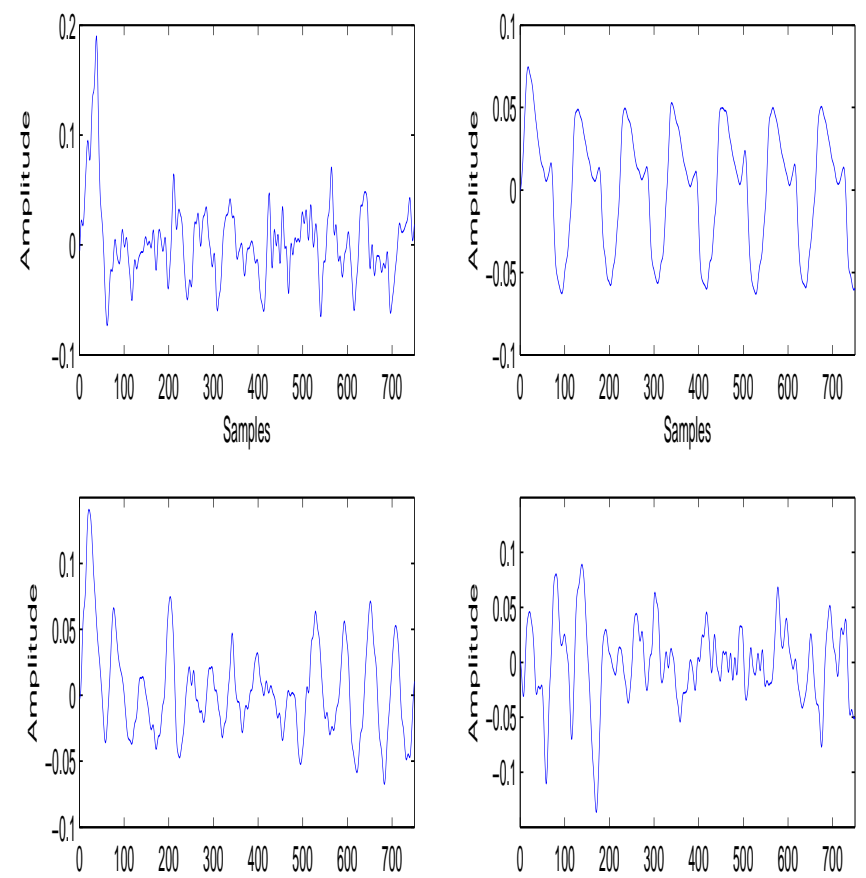

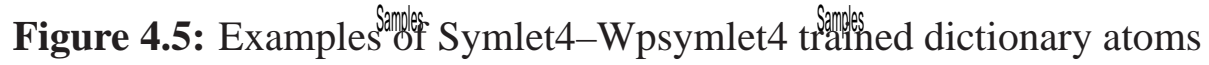




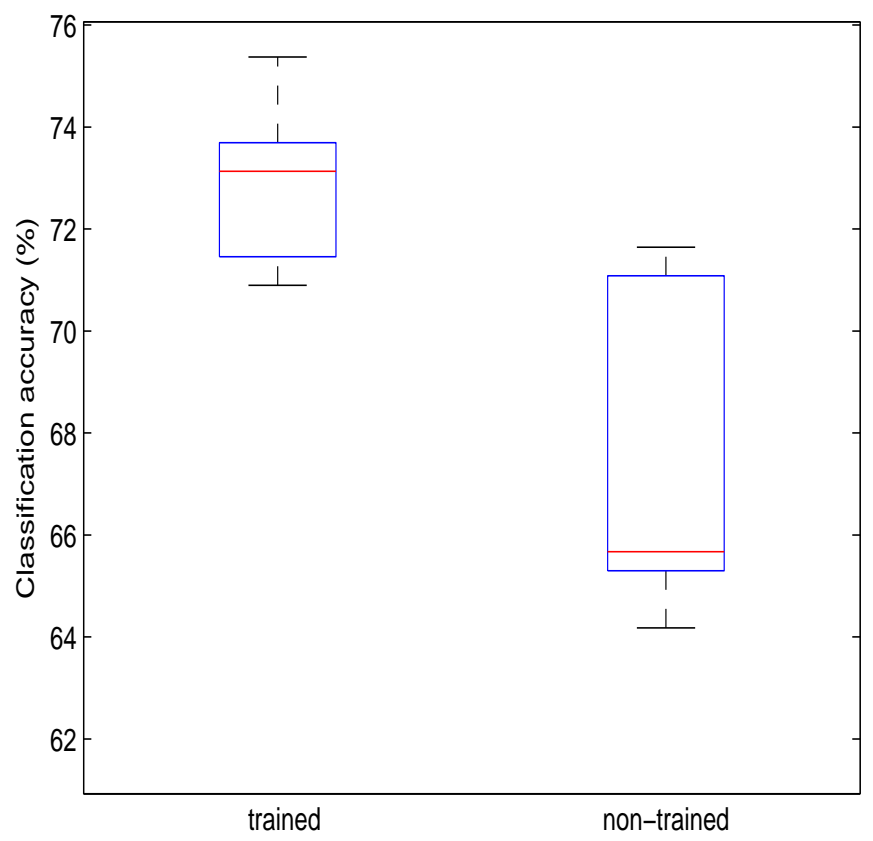

Figure 4.6: Boxplot of the classification accuracy of the five fold data sets of VF and VT using optimized trained and non-trained Gabor-Wpsymlet4 dictionaries

pend on the type of the dictionary that is used, but also on the size of the dictionary. Having a large dictionary can provide better classification accuracies, because more atoms would represent the different types of signals. Figure 4.8 can show that with the decrease of the size of the dictionary, the classification accuracy decreases. Increasing the size of the dictionary increases the computational time it takes to perform the LC-KSVD as shown in Figure 4.9, where the computational time of performing LC-KSVD on one fold was computed. Also the characterizing of the arrhythmias depends on what folds were used for training the dictionary.

A comparison on the performance of different types of classifiers such as linear, quadratic, and multi layer neural network, to classify the score feature of the LC-KSVD was performed. Figure 4.10 shows that there is no significant difference in using the quadratic classifier or a multi layer neural network classifier over a linear classifier to classify. 


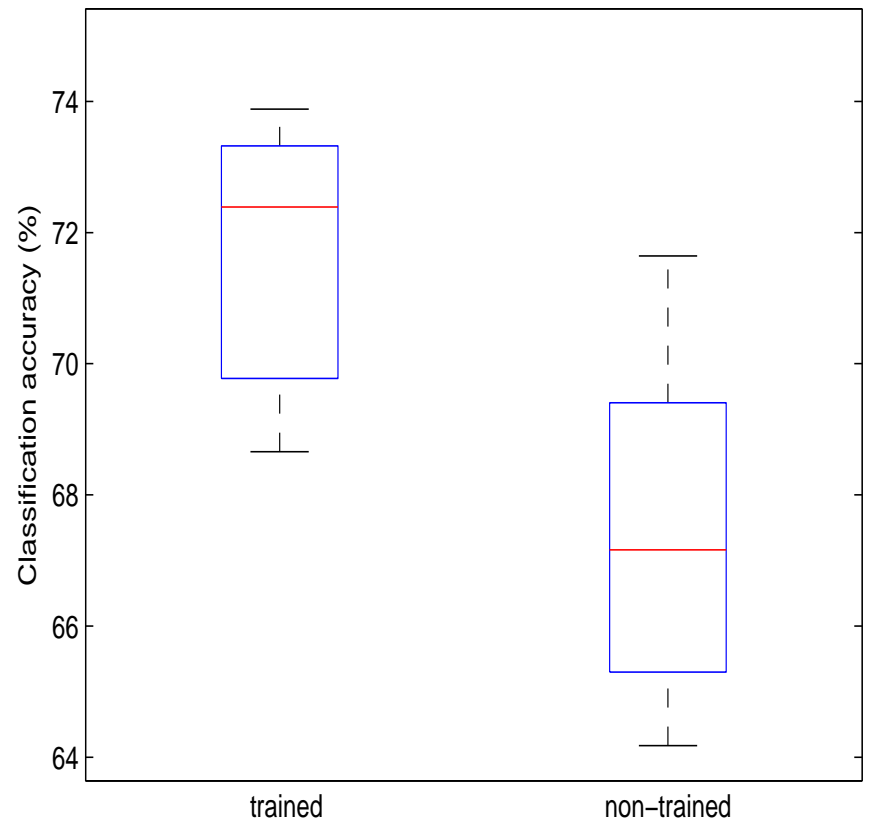

Figure 4.7: Boxplot of the classification accuracy of the five fold data sets of VF and VT using optimized trained and non-trained Gabor-Symlet4 dictionaries 


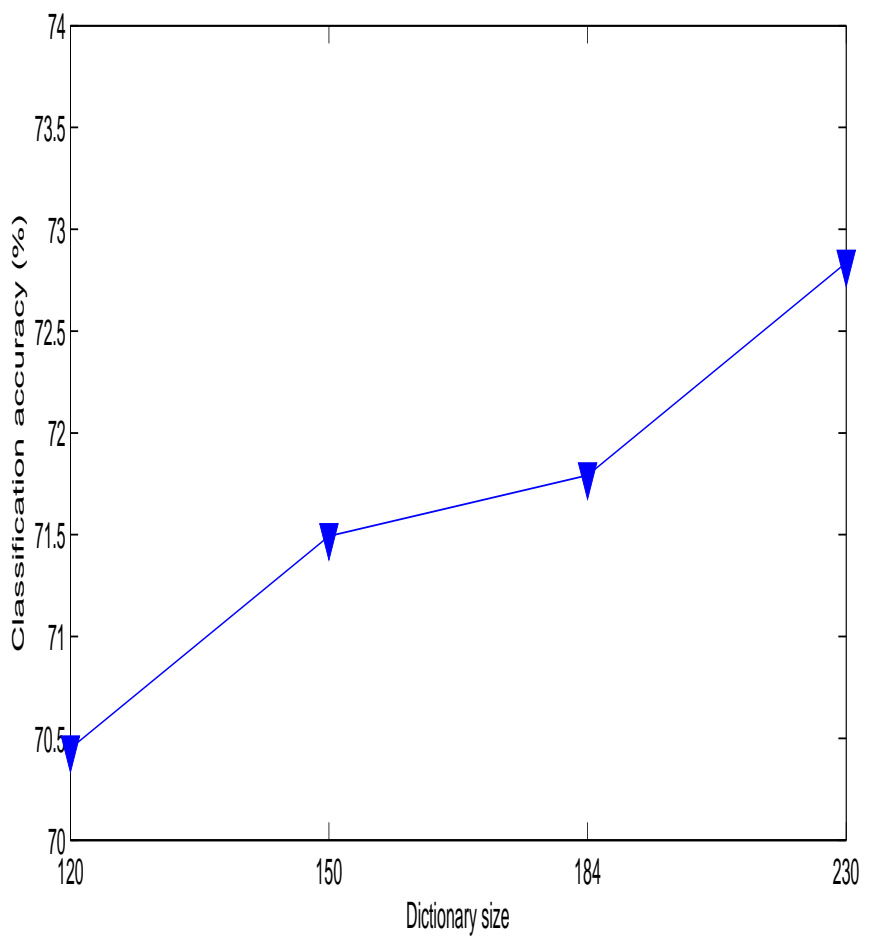

Figure 4.8: Classification accuracy using different sizes of the Gabor-Wpsym4 dictionary

\subsubsection{Results Using Full Dictionary}

Due to the limitation of having the number of the dictionary elements larger than the used database, the dictionary sizes were restricted to do the analysis. To overcome this and be able to use the entire dictionary an artificially derived database is generated from the original 670 segments by adding five different Gaussian white noise of $12,15,20,25$, and $30 \mathrm{~dB}$ to those segments. The resultant synthetic database consists of 3800 segments (1900 VF signal segments and 1900 VT signal segments) including the original 670 arrhythmic segments. After obtaining the new database the combinations of two dictionaries were chosen to be trained using the LC-KSVD with the same weight factors $\alpha=2$ and $\beta=0.01$, the sparsity factor $T=50$ and number of iterations (i.e., 2000 iterations). To validate the results a 5-fold cross validation was used, where the data-set was first randomly mixed up and then divided into five equal sets (760 segments each), and then the dictionaries were trained using four folds and tested with the remaining one. 


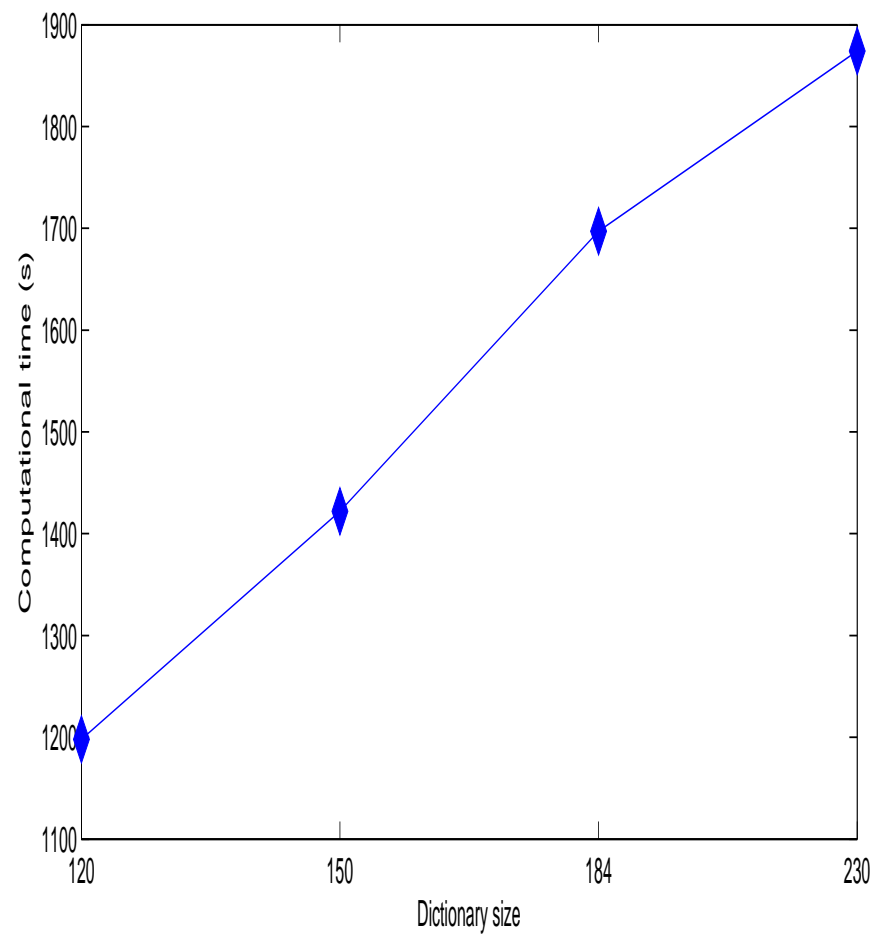

Figure 4.9: Computational time of one fold using LC-KSVD with respect to different sizes of the Gabor-Wpsym4 dictionary 


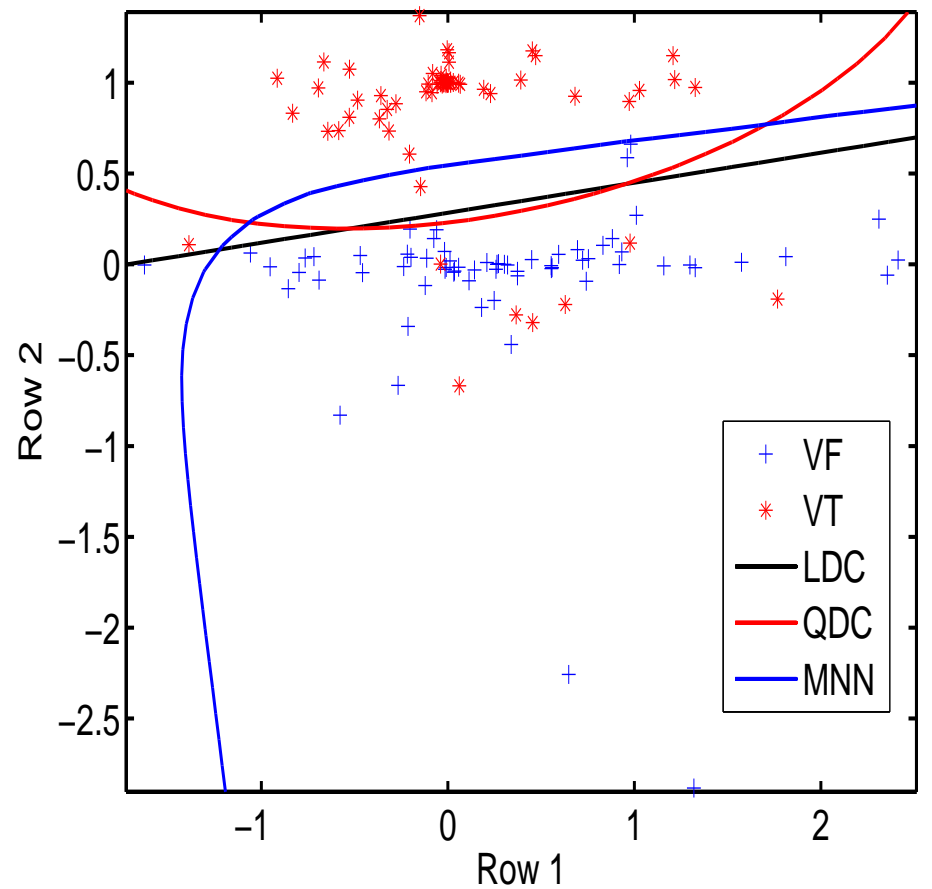

Figure 4.10: Comparing different types of classifiers for classifying the feature score of the LCKSVD 
Table 4.6 provide the classification accuracy of the three different dictionary combinations, where the Gabor-Symlet4 dictionary provide the highest classification accuracy of $87.4 \%$ compared to $86.2 \%$ and $87.0 \%$ of Gabor-Wpsymlet4 and Symlet4-Wpsymlet4 respectively. Using a full dictionary to characterize arrhythmic signals improved the classification accuracy, where characterizing VF signals was improved over the characterizing of VT signals, this is reflected in Tables 4.7 and 4.8 of the confusion matrix of classifying VF and VT signals using the learned dictionary Gabor-Wpsymlet4.

Table 4.9 shows the five fold classification accuracies of VF and VT signals with the Gabor-Wpsymlet4 wavelet hybrid dictionary with a total overall average classification accuracy of $\%$ 86.2. When the classification accuracy of tested signals is compared using a trained and nontrained dictionary, as expected the trained dictionary improves the accuracy over the non-trained one as shown in Figure 4.11, similarly when compared using the other two dictionaries such as Gabor-Symlet4 and Wpsymlet4-Symlet4.

Table 4.6: Average classification accuracy for different combinations of the full dictionaries for 2000 iterations of LC-KSVD

\begin{tabular}{|c|c|}
\hline Dictionary type combination & Classification accuracy (\%) \\
\hline Gabor-Symlet 4 & $87.4 \%$ \\
\hline Gabor-Wpsymlet4 & $86.2 \%$ \\
\hline Symlet4-Wpsymlet4 & $87.0 \%$ \\
\hline
\end{tabular}

Table 4.7: Confusion matrix of discriminating VF and VT signals using full Gabor-Wpsym4 dictionaries of LC-KSVD

\begin{tabular}{|c|c|c|c|c|}
\hline & & VF & VT & Total \\
\hline tested signals & VF & 358 & 22 & 380 \\
\hline & VT & 75 & 305 & 380 \\
\hline$\%$ & VF & $\mathbf{9 4 . 2}$ & 5.8 & 100 \\
\hline & VT & 19.7 & $\mathbf{8 0 . 3}$ & 100 \\
\hline
\end{tabular}


Table 4.8: Range of the 5-folds confusion matrix of discriminating VF and VT signals using full Gabor-Wpsym4 dictionary of LC-KSVD

\begin{tabular}{|c|c|}
\hline & Classification accuracy range \\
\hline VF & $90-97 \%$ \\
\hline VT & $70-84 \%$ \\
\hline
\end{tabular}

Table 4.9: The Classification accuracy for the four data sets using non-trained and LC-KSVD trained full Gabor-Wpsymlet4 dictionary

\begin{tabular}{|c|c|c|}
\hline Data Sets & Trained Dictionary (\%) & Untrained Dictionary (\%) \\
\hline fold 1 & $87.2 \%$ & $77.0 \%$ \\
\hline fold 2 & $87.2 \%$ & $77.2 \%$ \\
\hline fold 3 & $82.8 \%$ & $76.8 \%$ \\
\hline fold 4 & $85.8 \%$ & $78.2 \%$ \\
\hline fold 5 & $87.8 \%$ & $78.2 \%$ \\
\hline
\end{tabular}

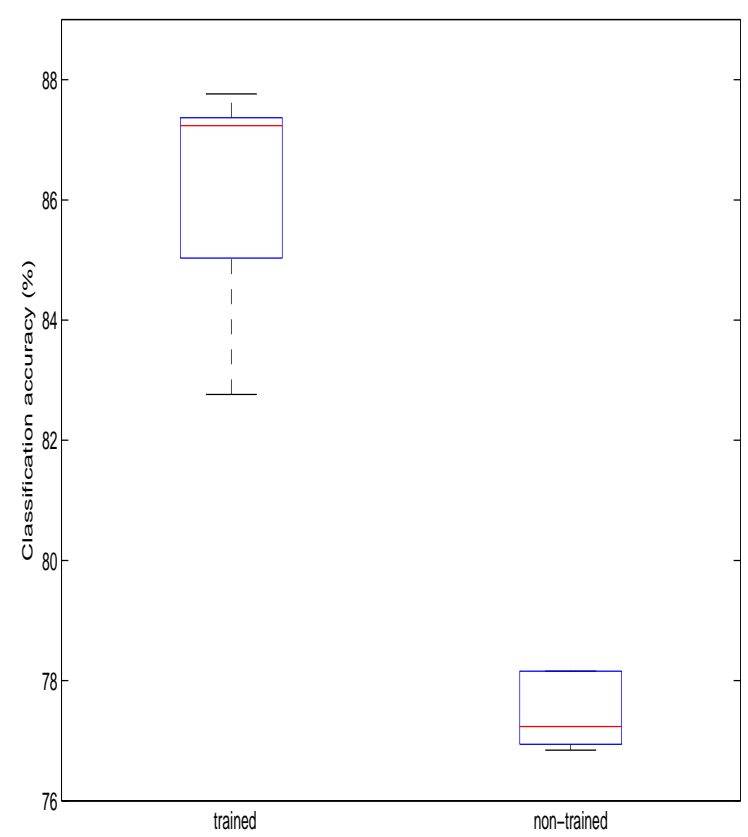

Figure 4.11: Boxplot of the classification accuracies of the five folds using trained and non-trained Gabor-Wpsymlet4 dictionary 


\subsection{Results Summary}

In the presented analysis, the highest classification accuracy of VF and VT signals obtained was 73.3\%, using the optimized Symlet4-Wpsymlet4 and a database of 670 segments. When the error classifier term was removed from the objective function the classification accuracy reduced from $\mathbf{7 3 . 3 \%}$ to $\mathbf{7 0 . 4 \%}$. The classification accuracy of trained dictionary was higher $\mathbf{7 3 . 3 \%}$ ) than the non-trained dictionaries $(\mathbf{6 7 . 5 \%})$ re-emphasizing the benefits of using a data adaptive dictionary learning approach in learning more discriminative information. As expected increasing the dictionary size increases the ability to accurately classify signals by improving the modeling flexibility but at the expense of computational time.

For the full dictionaries analysis using an artificial increased database from the actual data the highest classification accuracy of VF and VT signals was obtained using the Gabor-Symlet4 of $\mathbf{8 7 . 4 \%}$ compared to $\mathbf{8 7 . 0 \%}$ of Symlet4-Wpsymlet4, and $\mathbf{8 6 . 2 \%}$ Gabor-Wpsymlet4. Although higher classification accuracies were achieved using the artificially increased database, this may not reflect the true scenario as the added noise could have biased the signal morphologies more towards VF which might explain the higher classification accuracies of VF in comparison with VT. This exercise was to show that with increased database sizes we might have more flexibility in modeling these arrhtyhmias, however in reality obtaining vast databases for ventricular arrhythmias is a difficult challenge due to high mortality rates, ethical, and data privacy issues.

From the results section of this thesis, it can be concluded that the discriminative sparse coding using dictionary learning methods such as LC-KSVD does demonstrate high potential in successfully capturing the compact discriminative structures (time-frequency subspace) of ventricular arrhtyhmias. 


\subsection{Chapter 4 Summary}

This chapter presented the performance evaluation of the discriminative spare coding of ventricular arrhythmias using dictionary learning algorithm LC-KSVD. The chapter presents the results for different scenarios (hybrid dictionaries, LC-KSVD1, LC-KSVD2) and comparative performances between trained and non-trained approaches. The effect of dictionary size and the database sizes on the performance was also demonstrated. The chapter was concluded with a summary of results. 


\section{Chapter 5}

\section{Conclusions and Future Work}

\subsection{Summary and Conclusions}

In this thesis, automated learning of underlying discriminative structures of ventricular arrhythmias was investigated. A label consistent K-SVD dictionary learning algorithm was used to analyze ventricular fibrillation and ventricular tachycardia ECG segments using the discriminative sparse code obtained from a trained dictionary. A databases consisting of 670 arrhythmia segments from the publicly available MIT-BIH databases was used to train and classify using a predictive linear classifier. Optimal parameter choices for the analysis of ventricular arrhtyhmia were identified experimentally. Using these parameters, hybrid dictionaries were trained and their discriminative sparse codes were generated and these codes were validated for their discriminating ability. Three different hybrid optimized dictionaries: Gabor-Symlet4, Gabor-Wpsymlet4, and Symlet4-Wpsymlet4 achieved classification accuracies of $71.64 \%, 72.84 \%$, and $73.28 \%$ respectively.

The comparative results between trained and non-trained dictionaries validate the need for dictionary learning approaches in learning the underlying discriminative signal structures. Compact representation of these arrhythmias may lead to better characterization and insight into subclasses of these arrhythmias. This will also positively impact the intelligence of decision algorithms in implantable devices. From a mechanistic view point, these discriminative sparse code representations might reveal hidden information in the genesis of these arrhythmias. 


\subsection{Future Work}

Future work includes characterizing sub classes of arrhythmias such as polymorphic and monomorphic ventricular tachycardia, and organized and dis-organized ventricular fibrillation. In addition, with discriminative sparse coding we can identify sparse code templates for groups of arrhythmic signals which can be correlated to pathologies. Also, using these discriminative sparse code templates from multi-channel ECG recording it might be possible to infer regional variations in the arrhythmic activities. 


\section{Appendix A}

\section{Abbreviations}

ECG Electrocardiogram

VF Ventricular Fibrillation

VT Ventricular Tachycardia

MVT Monomorphic Ventricular Tachycardia

PVT Polymorphic Ventricular Tachycardia

VA Ventricular Activity

AA Atrial Activity

STFT Short Time Fourier Transform

CWT Continues Wavelet Transform

DWT Discrete Wavelet Transform

MP Matching Pursuit

OMP Orthogonal Matching Pursuit

K-SVD K-Singular Value Decomposition

LC-KSVD Label Consistent K-SVD

LDA Linear Discriminant Analysis

LDC Linear Discriminant Classifier

QDC Quadratic Discriminant Classifier

MNN Multi Layer Neural Network 


\section{Appendix B}

\section{Optimization Results}

\section{B.1 Sample optimization results using different $\alpha$ for a subset of data using Gabor and Sym4 dictionary}

Table B.1: Classification accuracy and error 1 for different $\alpha$ parameters for 2000 iterations of LC-KSVD

\begin{tabular}{|c|c|c|}
\hline$\alpha$ & Classification accuracy (\%) & Error 1 \\
\hline 0.01 & $64.18 \%$ & 0.004 \\
\hline 0.1 & $67.16 \%$ & 0.004 \\
\hline 0.5 & $69.40 \%$ & 0.002 \\
\hline 1 & $76.12 \%$ & 0.005 \\
\hline 2 & $78.36 \%$ & 0.008 \\
\hline 4 & $60.45 \%$ & 0.017 \\
\hline 6 & $61.19 \%$ & 0.023 \\
\hline
\end{tabular}

B.2 Sample optimization results using different $\alpha$ and $\beta$ for a subset of data using Gabor and Sym4 dictionary 
Table B.2: Total error 2 for different combinations of $\alpha$ and $\beta$ parameters for 2000 iterations of LC-KSVD

\begin{tabular}{|c|c|c|c|c|c|c|c|}
\hline & $\alpha=\mathbf{0 . 0 1}$ & $\alpha=\mathbf{0 . 1}$ & $\alpha=\mathbf{0 . 5}$ & $\alpha=\mathbf{1}$ & $\alpha=\mathbf{2}$ & $\alpha=\mathbf{4}$ & $\alpha=\mathbf{6}$ \\
\hline$\beta=\mathbf{0 . 0 1}$ & 0.0033 & 0.0037 & 0.0011 & 0.0045 & 0.0089 & 0.016 & 0.025 \\
\hline$\beta=\mathbf{0 . 1}$ & 0.0033 & 0.0033 & 0.0019 & 0.0051 & 0.0088 & 0.017 & 0.024 \\
\hline$\beta=\mathbf{0 . 5}$ & 0.0039 & 0.003 & 0.0016 & 0.0043 & 0.0092 & 0.015 & 0.028 \\
\hline$\beta=\mathbf{1}$ & 0.0042 & 0.0037 & 0.0018 & 0.0045 & 0.0094 & 0.016 & 0.024 \\
\hline$\beta=\mathbf{2}$ & 0.003 & 0.0025 & 0.0007 & 0.0046 & 0.0092 & 0.016 & 0.023 \\
\hline$\beta=\mathbf{4}$ & 0.002 & 0.0021 & 0.0017 & 0.0007 & 0.0088 & 0.016 & 0.024 \\
\hline$\beta=\mathbf{6}$ & 0.0017 & 0.0016 & 0.0019 & 0.0047 & 0.0091 & 0.016 & 0.022 \\
\hline
\end{tabular}

Table B.3: Classification accuracy for different combinations of $\alpha$ and $\beta$ parameters for 2000 iterations of LC-KSVD

\begin{tabular}{|c|c|c|c|c|c|c|c|}
\hline & $\alpha=\mathbf{0 . 0 1}$ & $\alpha=\mathbf{0 . 1}$ & $\alpha=\mathbf{0 . 5}$ & $\alpha=\mathbf{1}$ & $\alpha=\mathbf{2}$ & $\alpha=\mathbf{4}$ & $\alpha=\mathbf{6}$ \\
\hline$\beta=\mathbf{0 . 0 1}$ & $61.19 \%$ & $70.89 \%$ & $65.67 \%$ & $66.42 \%$ & $73.13 \%$ & $79.85 \%$ & $71.64 \%$ \\
\hline$\beta=\mathbf{0 . 1}$ & $68.66 \%$ & $67.16 \%$ & $73.13 \%$ & $63.43 \%$ & $68.66 \%$ & $77.61 \%$ & $62.69 \%$ \\
\hline$\beta=\mathbf{0 . 5}$ & $62.69 \%$ & $65.67 \%$ & $69.40 \%$ & $70.9 \%$ & $76.12 \%$ & $72.39 \%$ & $70.15 \%$ \\
\hline$\beta=\mathbf{1}$ & $61.194 \%$ & $64.92 \%$ & $74.63 \%$ & $72.39 \%$ & $73.89 \%$ & $79.10 \%$ & $65.67 \%$ \\
\hline$\beta=\mathbf{2}$ & $62.69 \%$ & $64.92 \%$ & $71.64 \%$ & $72.39 \%$ & $74.63 \%$ & $71.64 \%$ & $73.13 \%$ \\
\hline$\beta=\mathbf{4}$ & $64.92 \%$ & $75.73 \%$ & $65.67 \%$ & $71.64 \%$ & $75.37 \%$ & $72.39 \%$ & $70.9 \%$ \\
\hline$\beta=\mathbf{6}$ & $67.91 \%$ & $69.40 \%$ & $73.13 \%$ & $73.88 \%$ & $70.15 \%$ & $71.64 \%$ & $69.40 \%$ \\
\hline
\end{tabular}




\section{Bibliography}

[1] Stuart Ira Fox. Human physiology. McGraw-Hill Higher Education, 2008.

[2] Olivier Rioul and Martin Vetterli. Wavelets and Signal Processing. IEEE signal processing magazine, 8(LCAV-ARTICLE-1991-005):14-38, 1991.

[3] Douglas P. Zipes and Jose Jalife. Cardiac Electrophysiology: From Cell to Bedside. Elsevier Health Sciences, 2013.

[4] Heart and Stroke Foundation. Statistics. Last accessed May 29th, 2015.

[5] Michael A. Chen. Ventricular Tachycardia. A.D.A.M. Medical Encyclopedia, May 2014.

[6] Life In The Fastlane. ECG Library Ventricular Tachycardia. Last accessed September 10th, 2015.

[7] ACLS Algorthims. Other Tachycardia Rhythms. Last accessed June 18th,2015.

[8] Life In The Fastlane. ECG Library Polymorphic VT \& Torsades de Pointes (TdP). Last accessed September 10th, 2015.

[9] Life In The Fastlane. ECG Library Ventricular Fibrillation. Last accessed September 10th, 2015.

[10] Luis A Pires, Michael H Lehmann, Russell T Steinman, John J Baga, and Claudio D Schuger. Sudden Death in Implantable Cardioverter-Defibrillator Recipients: Clinical Context, Arrhythmic Events and Device Responses. Journal of the American College of Cardiology, 33(1):24-32, 1999. 
[11] Michael A. Chen. Ventricular Fibrillation. A.D.A.M. Medical Encyclopedia, May 2014.

[12] Markos G Tsipouras, Dirnitrois I Fotiadis, and D Sideris. Arrhythmia Classification Using the RR-interval Duration Signal. In Computers in Cardiology, pages 485-488. IEEE, 2002.

[13] Vessela Krasteva and Irena Jekova. Assessment of ECG Frequency and Morphology Parameters for Automatic Classification of Life-threatening Cardiac Arrhythmias. Physiological Measurement, 26(5):707, 2005.

[14] Nitish V Thakor, Yi-Sheng Zhu, and Kong-Yan Pan. Ventricular Tachycardia and Fibrillation Detection by a Sequential Hypothesis Testing Algorithm. Biomedical Engineering, IEEE Transactions on, 37(9):837-843, 1990.

[15] Nitish V Thakor, Ananth Natarajan, and Gordon F Tomaselli. Multiway Sequential Hypothesis Testing for Tachyarrhythmia Discrimination. Biomedical Engineering, IEEE Transactions on, 41(5):480-487, 1994.

[16] Muhammad Abdullah Arafat, Abdul Wadud Chowdhury, and Md Kamrul Hasan. A Simple Time Domain Algorithm for the Detection of Ventricular Fibrillation in Electrocardiogram. Signal, Image and Video Processing, 5(1):1-10, 2011.

[17] Robert D Throne, Janice M Jenkins, Lorenzo DiCarlo, et al. A Comparison of Four New Time-Domain Techniques for Discriminating Monomorphic Ventricular Tachycardia from Sinus Rhythm Using Ventricular Waveform Morphology. Biomedical Engineering, IEEE Transactions on, 38(6):561-570, 1991.

[18] Richard H Clayton, Alan Murray, and Ronald WF Campbell. Comparison of Four Techniques for Recognition of Ventricular Fibrillation from the Surface ECG. Medical and Biological Engineering and Computing, 31(2):111-117, 1993.

[19] Irena Jekova. Comparison Of Five Algorithms for the Detection of Ventricular Fibrillation from the Surface ECG. Physiological measurement, 21(4):429, 2000. 
[20] AJ Stewart, JD Allen, and AAJ Adgey. Frequency Analysis of Ventricular Fibrillation and Resuscitation Success. QJM, 85(1):761-769, 1992.

[21] Hans-Ulrich Strohmenger, Karl H Lindner, and Charles G Brown. Analysis of the Ventricular Fibrillation ECG Signal Amplitude and Frequency Parameters as Predictors of Countershock Success in Humans. CHEST Journal, 111(3):584-589, 1997.

[22] Trygve Eftestøl, Kjetil Sunde, Sven Ole Aase, John Håkon Husøy, and Petter Andreas Steen. Predicting Outcome of Defibrillation by Spectral Characterization and Nonparametric Classification of Ventricular Fibrillation in Patients with Out-of-hospital Cardiac Arrest. Circulation, 102(13):1523-1529, 2000.

[23] Markos G Tsipouras and Dimitrios I Fotiadis. Automatic Arrhythmia Detection Based on Time and Time-Frequency Analysis of Heart Rate Variability. Computer Methods and Programs in Biomedicine, 74(2):95-108, 2004.

[24] Valtino X Afonso and Willis J Tompkins. Detecting Ventricular Fibrillation. Engineering in Medicine and Biology Magazine, IEEE, 14(2):152-159, 1995.

[25] Krishnanand Balasundaram, Stephane Masse, Krishnakumar Nair, and Karthikeyan Umapathy. A Classification Scheme for Ventricular Arrhythmias Using Wavelets Analysis. Medical \& Biological Engineering \& Computing, 51(1-2):153-164, 2013.

[26] Krishnanand Balasundaram, Stephane Masse, Krishnakumar Nair, Talha Farid, Kumaraswamy Nanthakumar, and Karthikeyan Umapathy. Wavelet-Based Features for Characterizing Ventricular Arrhythmias in Optimizing Treatment Options. In Engineering in Medicine and Biology Society, EMBC, 2011 Annual International Conference of the IEEE, pages 969-972. IEEE, 2011.

[27] Hamid Khorrami and Majid Moavenian. A Comparative Study of DWT, CWT and DCT Transformations in ECG Arrhythmias Classification. Expert Systems with Applications, 37(8):5751-5757, 2010. 
[28] G Selvakumar, K Boopathy Bagan, and B Chidambararajan. Wavelet decomposition for detection and classification of critical ecg arrhythmias. In Proceeding of the 8th WSEAS International Conference on Mathematics and computers in Biology and Chemistry, pages 80-84, 2007.

[29] Krishnanand Balasundaram. Analysis of Electrocardiograms During Human Ventricular Arrhythmias for Optimizing Treatment Options. 2012.

[30] Molinero J Hernandez. Sparse decompositions for ventricular and atrial activity separation. In MS thesis, Signal Processing Institute, Ecole Polytechnique Fédérale de. Citeseer, 2005.

[31] Divorra O Escoda, Lorenzo Granai, Mathieu Lemay, Molinero J Hernandez, Pierre Vandergheynst, and Jean-Marc Vesin. Ventricular and Atrial Activity Estimation Through Sparse ECG Signal Decompositions. In Acoustics, Speech and Signal Processing, 2006. ICASSP 2006 Proceedings. 2006 IEEE International Conference on, volume 2, pages II-II. IEEE, 2006.

[32] Ivaylo Christov, Gèrman Gómez-Herrero, Vessela Krasteva, Irena Jekova, Atanas Gotchev, and Karen Egiazarian. Comparative Study of Morphological and Time-Frequency ECG Descriptors for Heartbeat Classification. Medical engineering \& physics, 28(9):876-887, 2006.

[33] Alexandros Pantelopoulos and Nikolaos Bourbakis. ECG Beat Classification Using Optimal Projections in Overcomplete Dictionaries. In Tools with Artificial Intelligence (ICTAI), 2011 23rd IEEE International Conference on, pages 1099-1105. IEEE, 2011.

[34] Boris Mailhé, Rémi Gribonval, Frédéric Bimbot, Mathieu Lemay, Pierre Vandergheynst, and Jean-Marc Vesin. Dictionary Learning for the Sparse Modelling of Atrial Fibrillation in ECG Signals. In Acoustics, Speech and Signal Processing, 2009. ICASSP 2009. IEEE International Conference on, pages 465-468. IEEE, 2009.

[35] Seung Jae Lee, Jun Luan, and Pai H Chou. ECG Signal Reconstruction from Undersampled Measurement Using a Trained Overcomplete Dictionary. 2014. 
[36] Chunguang Wang, Jinjiang Liu, and Jixiang Sun. Compression Algorithm for Electrocardiograms Based on Sparse Decomposition. Frontiers of Electrical and Electronic Engineering in China, 4(1):10-14, 2009.

[37] Sherin M Mathews, Luisa F Polania, and Kenneth E Barner. Leveraging a Discriminative Dictionary Learning Algorithm for Single-lead ECG Classification. In Biomedical Engineering Conference (NEBEC), 2015 41st Annual Northeast, pages 1-2. IEEE, 2015.

[38] Iman Kalaji, Krishnand Balasundaram, and Karthikeyan Umapathy. Discriminative Sparse Coding of ECG During Ventricular Arrhythmias Using LC-K-SVD Approach. In Engineering in Medicine and Biology Society, EMBC, 2015 Annual International Conference of the IEEE, pages 5211-5214. IEEE, 2015.

[39] Robi Polikar. The Engineer's Ultimate Guide to Wavelet Analysis-The Wavelet Tutorial. Available at http://www. public. iastate. edu/ rpolikar/WAVELETS/WTtutorial. html, 1996.

[40] Stéphane G Mallat and Zhifeng Zhang. Matching Pursuits with Time-Frequency Dictionaries. Signal Processing, IEEE Transactions on, 41(12):3397-3415, 1993.

[41] Tony T Cai and Lie Wang. Orthogonal Matching Pursuit for Sparse Signal Recovery with Noise. Information Theory, IEEE Transactions on, 57(7):4680-4688, 2011.

[42] Raazia Mazhar. Optimized Dictionary Design and Classification Using the Matching Pursuits Dissimilarity Measure. 2009.

[43] Michal Aharon, Michael Elad, and Alfred Bruckstein. K-SVD: An Algorithm for Designing Overcomplete Dictionaries for Sparse Representation. Signal Processing, IEEE Transactions on, 54(11):4311-4322, 2006.

[44] Michael S Lewicki and Bruno A Olshausen. Probabilistic Framework for the Adaptation and Comparison of Image Codes. JOSA A, 16(7):1587-1601, 1999. 
[45] Kjersti Engan, Sven Ole Aase, and J Hakon Husoy. Method of Optimal Directions for Frame Design. In Acoustics, Speech, and Signal Processing, 1999. Proceedings., 1999 IEEE International Conference on, volume 5, pages 2443-2446. IEEE, 1999.

[46] Zhuolin Jiang, Zhe Lin, and Larry S Davis. Label Consistent K-SVD: Learning a Discriminative Dictionary for Recognition. Pattern Analysis and Machine Intelligence, IEEE Transactions on, 35(11):2651-2664, 2013.

[47] Zhuolin Jiang, Zhe Lin, and Larry S Davis. Learning a Discriminative Dictionary for Sparse Coding Via Label Consistent K-SVD. In Computer Vision and Pattern Recognition (CVPR), 2011 IEEE Conference on, pages 1697-1704. IEEE, 2011.

[48] Richard O Duda, Peter E Hart, and David G Stork. Pattern Classification. John Wiley \& Sons, 2012.

[49] Ary L Goldberger, Luis AN Amaral, Leon Glass, Jeffrey M Hausdorff, Plamen Ch Ivanov, Roger G Mark, Joseph E Mietus, George B Moody, Chung-Kang Peng, and H Eugene Stanley. Physiobank, Physiotoolkit, and Physionet Components of a New Research Resource for Complex Physiologic Signals. Circulation, 101(23):e215-e220, 2000.

[50] JA Van Alste and TS Schilder. Removal of Base-line Wander and Power-line Interference from the ECG by an Efficient FIR Filter with a Reduced Number of Taps. Biomedical Engineering, IEEE Transactions on, (12):1052-1060, 1985.

[51] Monica Fira and Liviu Goras. Biomedical Signal Compression Based on Basis Pursuit. In Proceedings of the 2009 International Conference on Hybrid Information Technology, pages 541-545. ACM, 2009.

[52] Chia-Hung Lin. Frequency-Domain Features for ECG Beat Discrimination Using Grey Relational Analysis-Based Classifier. Computers \& Mathematics with Applications, 55(4):680_ 690, 2008. 
[53] Kei-ichiro Minami, Hiroshi Nakajima, and Takeshi Toyoshima. Real-Time Discrimination of Ventricular Tachyarrhythmia with Fourier-Transform Neural Network. Biomedical Engineering, IEEE Transactions on, 46(2):179-185, 1999.

[54] Labib Khadra, Amjed Al-Fahoum, and Saeed Binajjaj. A New Quantitative Analysis Technique for Cardiac Arrhythmia Using Bispectrum and Bicoherency. In Engineering in Medicine and Biology Society, 2004. IEMBS'04. 26th Annual International Conference of the IEEE, volume 1, pages 13-16. IEEE, 2004.

[55] Mathworks. Wavelet Toolbox User's Guide. Last accessed June 25th,2015.

[56] Ingrid Daubechies et al. Ten Lectures on Wavelets, volume 61. SIAM, 1992.

[57] Mohammad H Kadbi, Javad Hashemi, Hamid R Mohseni, and Arash Maghsoudi. Classification of ECG Arrhythmias Based on Statistical and Time-Frequency Features. 2006.

[58] Abibullaev Berdakh and Hee-Don Seo. A New QRS Detection Method Using Wavelet and Artificial Neural Networks. J Med Syst. DOI, 10. 